\title{
Voronoi summation formulae and multiplicative functions on permutations
}

\author{
Vytas Zacharovas* \\ Department of Mathematics and Informatics \\ Vilnius University \\ Naugarduko 24, Vilnius \\ Lithuania
}

June 24, 2018

\begin{abstract}
We prove a Tauberian theorem for the Voronoi summation method of divergent series with an estimate of the remainder term. The results on the Voronoi summability are then applied to analyze the mean values of multiplicative functions on random permutations.
\end{abstract}

Key words: Tauberian theorems, divergent series, Voronoi summability, Nörlund summability, symmetric group, random permutations, additive functions, multiplicative functions, Berry-Esseen bound.

\section{Introduction}

\subsection{Tauberian theorem for Voronoi summation method}

The classical result of Abel states that if an infinite series $\sum_{n=0}^{\infty} a_{n}$ converges and its sum is equal to $A$ then there exists the limit

$$
\lim _{x \nearrow 1} \sum_{n=0}^{\infty} a_{n} x^{n}=A .
$$

*This paper is based on chapters 1.1 and 1.2 of author's doctorial dissertation that was written and defended at Vilnius University in 2004 under supervision of prof. E. Manstavičius. The final version of the paper was written during the authors work at Institute of Statistical Science, Academia Sinica (Taiwan). 
The converse statement is not true, as can be seen by considering the series $\sum_{n=0}^{\infty}(-1)^{n}$, which is divergent in spite of existence of the $\operatorname{limit}_{x} \lim _{x 1} \sum_{n=0}^{\infty}(-1)^{n} x^{n}=$ $1 / 2$. Tauber [13] in 1897 proved that if in addition to existence of the limit (1) the coefficients of the infinite series satisfy condition

$$
\sum_{k=0}^{n} k a_{k}=o(n)
$$

as $n \rightarrow \infty$, then the series $\sum_{n=0}^{\infty} a_{n}$ converges and its sum is equal to $A$. Moreover the Tauber's condition (2) is in fact necessary for the convergence of the series. This Tauber's result has given rise to the whole class of so called Tauberian theorems. See book [7] for the review of the subject.

It can be shown that Tauber's condition (2) imposed on coefficients $a_{j}$ alone is enough to provide an asymptotic estimate for partial sums of the formal series

$$
\sum_{k=0}^{n} a_{k}=g\left(e^{-1 / n}\right)+o(1)
$$

where $g(z)=\sum_{j=0}^{\infty} a_{j} z^{j}$. Note that here $g\left(e^{-1 / n}\right)$ does not need to have a limit as $n \rightarrow \infty$.

Voronoi (the same summation method has been later reintroduced by Nörlund and is often named him) introduced a summation method of divergent series which is defined by a sequence of non-negative numbers $w_{j} \geqslant 0$, that are not identically equal to zero. Suppose $\sum_{k=0}^{\infty} a_{k}$ is a formal series. If there exists a limit

$$
\lim _{n \rightarrow \infty} \frac{w_{0} s_{n}+w_{1} s_{n-1}+\cdots+w_{n} s_{0}}{w_{0}+w_{1}+\cdots+w_{n}}=s \in \mathbb{C},
$$

where $s_{k}=a_{0}+a_{1}+\cdots+a_{k}$, then we say that the series $\sum_{k=0}^{\infty} a_{k}$ can be summed in the sense of Voronoi and its Voronoi sum is equal to $s$. In such case we write

$$
\left(W, w_{n}\right) \sum_{k=0}^{\infty} a_{k}=s .
$$

If, for example, we take $w_{0}=1$ and $w_{j}=0, j \geqslant 1$ then the Voronoi summation for such choice of $w_{j}$ will coincide with the usual definition of convergence of an infinite series. The choice $w_{j} \equiv 1$ leads to the definition of Cesàro $(C, 1)$ summability. We refer the reader to the classical book of Hardy [6] for more examples and discussions on the subject of divergent series.

Note that the weighted average of partial sums of the formal series $\sum_{j=0}^{\infty} a_{j}$ defining Voronoi summation method can be rewritten as

$$
\frac{w_{0} s_{n}+w_{1} s_{n-1}+\cdots+w_{n} s_{0}}{w_{0}+w_{1}+\cdots+w_{n}}=\frac{1}{W_{n}} \sum_{k=0}^{n} a_{k} W_{n-k}
$$


where $W_{j}=w_{0}+w_{1}+\cdots+w_{j}$ are positive numbers. In what follows we will refer to the above weighted average of partial sums $s_{j}$ as Voronoi mean. Thus given a fixed sequence of positive numbers $W_{j}$, a natural question arises, what would be the generalization of the classical Tauber's condition (2) on $a_{k}$ that would imply the following asymptotic for Voronoi mean

$$
\frac{1}{W_{n}} \sum_{k=0}^{n} a_{k} W_{n-k}=g\left(e^{-1 / n}\right)+o(1),
$$

as $n \rightarrow \infty$, where as before $g(x)=\sum_{k=0}^{\infty} a_{k} x^{k}$ ? We provide a partial answer to this question for the the class of sequences $W_{j}$ whose generating function is of the form

$$
\sum_{j=0}^{\infty} W_{j} z^{j}=\exp \left\{\sum_{k=1}^{\infty} \frac{u_{k}}{k} z^{k}\right\}
$$

with $0<u^{-} \leqslant u_{k} \leqslant u^{+}<\infty$. We will show that for this class of Voronoi methods, if $a_{k}$ satisfy condition

$$
\frac{1}{W_{n}} \sum_{k=0}^{n} k a_{k} W_{n-k}=o(n)
$$

as $n \rightarrow \infty$, then the asymptotic (3) for Voronoi means holds. Note that the sequence $W_{n}$ satisfying our condition imposed on the form of its generating function does not need to be increasing, unlike the sequences arising from the definition of Voronoi summation, in which case $W_{n}$ as a partial sum of $w_{j}$ should be increasing $W_{n+1}-W_{n}=w_{n+1} \geqslant 0$. This class is large enough to contain the class of Cesàro methods with parameter $\theta>-1$ (see [6] for definition). An open question remains how far can we expand the class of Voronoi methods so that the condition (4) on $a_{n}$ would guarantee the asymptotic (3) for Voronoi means.

The central part of our argument is the inequality of the following theorem that allows us to estimate the error term in the asymptotic of Voronoi means (3) in terms of sums $\sum_{k=0}^{n} k a_{k} W_{n-k}$.

Theorem 1. Suppose $g(z)=\sum_{n=0}^{\infty} a_{n} z^{n}$ is an analytic function for $|z|<1$ and $p_{j}$ is a sequence of positive numbers that is defined by means of its generating function

$$
p(z)=\sum_{j=0}^{\infty} p_{j} z^{j}=\exp \left\{\sum_{k=1}^{\infty} \frac{d_{k}}{k} z^{k}\right\},
$$

where $d_{j}$ are positive numbers bounded from above and below $0<d^{-} \leqslant d_{j} \leqslant d^{+}$. Then there exists a positive constant $c=c\left(d^{+}, d^{-}\right)$, which depends on $d^{+}$and $d^{-}$ 
only, such that for all $n \geqslant 1$ holds the inequality

$$
\begin{aligned}
& \left|\frac{1}{p_{n}} \sum_{k=0}^{n} a_{k} p_{n-k}-g\left(e^{-1 / n}\right)-\frac{S(g ; n)}{n p_{n}}\right| \\
& \quad \leqslant c\left(\frac{1}{n^{\theta}} \sum_{j=1}^{n} \frac{|S(g ; j)|}{p_{j}} j^{\theta-2}+\frac{1}{p\left(e^{-1 / n}\right)} \sum_{j>n} \frac{|S(g ; j)|}{j} e^{-j / n}\right),
\end{aligned}
$$

where

$$
S(g ; j)=\sum_{k=0}^{j} a_{k} k p_{j-k}
$$

and $\theta=\min \left\{d^{-}, 1\right\}$.

A simple consequence of the above theorem is the direct generalization of Tauber's theorem for our class of Voronoi summation methods.

Theorem 2. Let $g(z)$ and $p(z)$ be the same as in Theorem 1 Then the relation

$$
\lim _{n \rightarrow \infty} \frac{1}{p_{n}} \sum_{k=0}^{n} a_{k} p_{n-k}=A \in \mathbb{C}
$$

holds if and only if the following two conditions are satisfied:

1 .

$$
\lim _{x \uparrow 1} g(x)=A,
$$

2.

$$
\sum_{k=0}^{n} a_{k} k p_{n-k}=o\left(n p_{n}\right) \quad \text { as } \quad n \rightarrow \infty
$$

\subsection{Application to analysis of generating functions}

In what follows, for any analytic function $H(z)=\sum_{j=0}^{\infty} H_{j} z^{j}$ we will denote by $\left[z^{n}\right] H(z)$ its $n$-th Taylor coefficient $H_{n}$. We can apply our results on Voronoi summation method to analysis of generating functions in the following way. Suppose we want to analyze the asymptotic behavior of the coefficients $\left[z^{n}\right] F(z)$ in the Taylor expansion of the generating function $F(z)$, which is analytic in the unit disc $|z|<1$. Suppose we can decompose the generating function $F(z)$ as a product of two functions

$$
F(z)=W(z) g(z)
$$


where $W(z)=\sum_{j=0}^{\infty} W_{j} z^{j}$ is a function with positive Taylor coefficients $W_{j}>0$ for $j \geqslant 1$ with $W_{0}=1$ and such that the coefficients in the Taylor expansion of its logarithmic derivative are bounded from above and below

$$
0<u^{-} \leqslant\left[z^{n}\right] \frac{W^{\prime}(z)}{W(z)} \leqslant u^{+}
$$

for all $n \geqslant 0$ by some fixed positive constants $u^{+}, u^{-}$. If in addition to the above restrictions on $W(z)$, our Tauberian condition (4), expressed in terms of generating functions as

$$
\left[z^{n-1}\right] W(z) g^{\prime}(z)=o\left(n W_{n}\right)
$$

is satisfied, then by the inequality (5) of Theorem 1 we get an estimate for the Taylor coefficients

$$
\left[z^{n}\right] F(z)=\left[z^{n}\right] W(z) g(z)=W_{n}\left(g\left(e^{-1 / n}\right)+o(1)\right),
$$

as $n \rightarrow \infty$. This approach can be compared with the other standard technique for analyzing asymptotic behavior of the Taylor coefficients of analytic functions. It is based on representing $\left[z^{n}\right] F(z)$ as a Cauchy integral

$$
\left[z^{n}\right] F(z)=\frac{1}{2 \pi i} \int_{|z|=r} \frac{F(z)}{z^{n+1}} d z .
$$

The further analysis depends on the amount and the type of information that we have on the behavior of $F(z)$ near its singularities and whether or not function $F(z)$ can be analytically extended to some area beyond the radius of convergence of its Taylor series. Flajolet and Odlyzko [3] analyzed the case when $F(z)$ can be decomposed as $F(z)=W(z) g(z)$ where $W(z)=\frac{1}{(1-z)^{\theta}}=\sum_{n=0}^{\infty}\left(\begin{array}{c}n+\theta-1 \\ n\end{array}\right) z^{n}$ and $g(z)$ is an analytic function in the circle $|z|<1+\varepsilon$, where $\varepsilon>0$. They proved that

$$
\left[z^{n}\right] \frac{1}{(1-z)^{\theta}} g(z)=\left(\begin{array}{c}
n+\theta-1 \\
n
\end{array}\right) g(1)\left(1+O\left(n^{-1}\right)\right) .
$$

The analysis of the Cauchy integral (8) usually becomes considerably more difficult if we do not know anything about the analytic extension of generating function $F(z)$ beyond the unit circle $|z|<1$. This is exactly the case with the generating function of the mean values of multiplicative functions on permutations, which is the main object of application of our theorems for Voronoi sums. Manstavičius in a series of papers [8], [9], [10] and [11] used a technique based on Halász'es [5] ideas for investigating asymptotic behavior of such Cauchy integrals. See also [1] for a modified version of this approach. The approach we use here exploits the fact that in the case of random permutations, the generating functions we consider are such that a simple upper bound can be obtained 
for the quantity $\left[z^{n-1}\right] W(z) g^{\prime}(z)$ when $W(z) g(z)$ is the appropriate decomposition of the generating function, thus immediately leading to the asymptotic of type $\left[z^{n}\right] W(z) g(z)=W_{n}\left(g\left(e^{-1 / n}\right)+o(1)\right)$. The advantage of such an approach is that it allows us to avoid the analysis of function $F(z)$ for complex values of $z$, which is particulary hard to do since such analysis requires estimating certain complicated trigonometric sums. We only use the information on the behavior of $g(x)$ for the real values $x$ that are close to 1 . The same approach has already been used in our papers [14] and [15] to analyze the distribution of additive and multiplicative functions with respect to Ewens measure.

\subsection{Random permutations}

Let $S_{n}$ be the symmetric group. Recall that $S_{n}$ is composed of all possible functions that bijectively map the set of first $n$ integers $\{1,2, \ldots, n\}$ into itself. Such functions are also called permutations. Every permutation $\sigma$ belonging to the symmetric group $S_{n}$ can be represented as an oriented graph, containing $n$ vertices that are labeled by natural numbers $1,2, \ldots, n$, and $n$ edges, each edge corresponding to a pair $(j, \sigma(j))$, starting at vertex $j$ and pointing to vertex $\sigma(j)$. Such graphs are characterized by the property that every edge has one and only one outgoing edge and one and only one incoming edge. It is easy to realize that each such a graph consists only of cyclical components. For example, let us consider permutation

$$
\lambda=\left(\begin{array}{ccccccccccccccc}
1 & 2 & 3 & 4 & 5 & 6 & 7 & 8 & 9 & 10 & 11 & 12 & 13 & 14 & 15 \\
4 & 2 & 9 & 6 & 7 & 10 & 8 & 5 & 3 & 1 & 11 & 12 & 15 & 13 & 14
\end{array}\right)
$$

belonging to $S_{15}$, written in its usual representation as a table consisting of two rows. The upper row contains the numbers $1,2, \ldots, 15$ and the lower row consists of their images $\lambda(1), \lambda(2), \ldots, \lambda(15)$. Such permutation corresponds to the graph consisting of seven cyclical components.

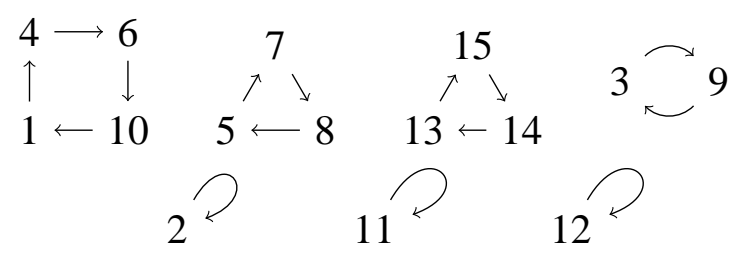

Following [11] we will consider the classes of additive and multiplicative functions on permutations whose values are determined by the decomposition of permutations into cyclical components. These functions are defined as follows. Suppose we have $n$ real numbers $\hat{h}(1), \hat{h}(2), \ldots, \hat{h}(n)$, then for each permutation 
$\sigma \in S_{n}$ we can compose a sum $h(\sigma)$ over all cycles in the graph of $\sigma$ so that for each cycle of length $j$ we add one summand $\hat{h}(j)$. Or equivalently,

$$
h(\sigma)=\hat{h}(1) \alpha_{1}(\sigma)+\hat{h}(2) \alpha_{2}(\sigma)+\cdots+\hat{h}(n) \alpha_{n}(\sigma),
$$

where $\alpha_{j}(\sigma)$ is the number of cycles of length $j$ in permutation $\sigma$. For example 15 numbers $\hat{h}(1), \hat{h}(2), \ldots, \hat{h}(15)$ will completely define the value of additive function $h(\sigma)$ on all $\sigma \in S_{15}$. In particular, our permutation $\lambda \in S_{15}$ contains one cycle of length 4 , two cycles of length 3 , one cycle of length 2 and three cycles of length 1 , therefore

$$
h(\lambda)=3 \hat{h}(1)+\hat{h}(2)+2 \hat{h}(3)+\hat{h}(4) .
$$

A simple example of an additive function is obtained if all $\hat{h}(j)$ are equal to 1 . The resulting additive function $w(\sigma)$ is then equal to the total number of cycles of in the graph of $\sigma$. For our example (9) we have $w(\lambda)=7$. Goncharov [4] was the first to analyze the limit distribution of $w(\sigma)$. He proved that the number of permutations $\sigma \in S_{n}$ satisfying inequality $\frac{w(\sigma)-\log n}{\sqrt{\log n}}<x$ divided by the total number of permutations $\left|S_{n}\right|=n$ ! converges to $\frac{1}{\sqrt{2 \pi}} \int_{-\infty}^{x} e^{-u^{2} / 2} d u$ as $n \rightarrow \infty$.

In a similar way we define multiplicative functions on the symmetric group $S_{n}$. Suppose we are given $n$ complex numbers $\hat{f}(1), \hat{f}(2), \ldots, \hat{f}(n)$. Then for each permutation $\sigma \in S_{n}$ we can assign a product $f(\sigma)$ over all cycles belonging to the oriented graph of $\sigma$ that contains one multiplicand $\hat{f}(j)$ corresponding to every cycle of size $j$ belonging to $\sigma$. In other words

$$
f(\sigma)=\hat{f}(1)^{\alpha_{1}(\sigma)} \hat{f}(2)^{\alpha_{2}(\sigma)} \cdots \hat{f}(n)^{\alpha_{n}(\sigma)},
$$

where we assume $0^{0}=1$. For our example (9) of $\lambda \in S_{15}$ we have

$$
f(\lambda)=\hat{f}(1)^{3} \hat{f}(2) \hat{f}(3)^{2} \hat{f}(4) .
$$

Suppose $d(\sigma)$ is a non-negative multiplicative function, which is not identically equal to zero. Then we can define a probabilistic measure $\nu_{n, d}$ on $S_{n}$ by the formula

$$
\nu_{n, d}(\sigma)=\frac{d(\sigma)}{\sum_{\tau \in S_{n}} d(\tau)} .
$$

The simplest and the most natural choice is to put $\hat{d}(j) \equiv 1$, which leads to the uniform probability measure

$$
\nu_{n}^{(1)}(\sigma)=\frac{1}{n !} .
$$

Thus Goncharov's result can be expressed in probabilistic terms as a limit theorem

$$
\nu_{n}^{(1)}\left(\frac{w(\sigma)-\log n}{\sqrt{\log n}}<x\right) \rightarrow \frac{1}{\sqrt{2 \pi}} \int_{-\infty}^{x} e^{-u^{2} / 2} d u, \quad \text { as } \quad n \rightarrow \infty,
$$


stating that the number of cycles $w(\sigma)$ in permutation $\sigma$ chosen with equal probability among all the permutations of the symmetric group $S_{n}$ is asymptotically normally distributed.

More generally if all $\hat{d}(j)$ are equal $\hat{d}(j) \equiv \theta>0$ then $d(\sigma)=\theta^{w(\sigma)}$, thus we obtain the so called Ewens probability measure

$$
\nu_{n}^{(\theta)}(\sigma)=\frac{\theta^{w(\sigma)}}{\sum_{\tau \in S_{n}} \theta^{\omega(\tau)}}=\frac{\theta^{w(\sigma)}}{\theta(\theta+1) \cdots(\theta+n-1)} .
$$

Let us denote by $M_{n}^{d}(f)$ a weighted mean of a multiplicative function $f: S_{n} \rightarrow \mathbb{C}$ with respect to the measure $\nu_{n, d}(\sigma)$ :

$$
M_{n}^{d}(f)=\sum_{\sigma \in S_{n}} f(\sigma) \nu_{n, d}(\sigma)=\frac{\sum_{\sigma \in S_{n}} f(\sigma) d(\sigma)}{\sum_{\sigma \in S_{n}} d(\sigma)} .
$$

In 2002 Manstavičius proved the following result.

Theorem A ([10]). Let $f: S_{n} \rightarrow \mathbb{C}$ be a multiplicative function, such that $|f(\sigma)| \leqslant 1$, satisfying the conditions:

$$
\sum_{j \leqslant n} \frac{1-\Re \hat{f}(j)}{j} \leqslant D
$$

and

$$
\frac{1}{n} \sum_{j=1}^{n}|\hat{f}(j)-1| \leqslant \mu_{n}=o(1)
$$

for some positive constant $D$ and some sequence $\mu_{n}$.

Suppose that the measure defining multiplicative function $d(\sigma)$ satisfies the condition $0<d^{-} \leqslant \hat{d}(j) \leqslant d^{+}$for all $j \geqslant 1$, with some fixed positive constants $d^{-}$and $d^{+}$, then there exist positive constants $c_{1}=c_{1}\left(d^{-}, d^{+}\right)$and $c_{2}=$ $c_{2}\left(d^{-}, d^{+}\right)$such that

$$
M_{n}^{d}(f)=\exp \left\{\sum_{j \leqslant n} \hat{d}(j) \frac{\hat{f}(j)-1}{j}\right\}+O\left(\mu_{n}^{c_{1}}+\frac{1}{n^{c_{2}}}\right) .
$$

We prove the following result.

Theorem 3. Let $f: S_{n} \rightarrow \mathbb{C}$ be a multiplicative function satisfying the condition $|f(\sigma)| \leqslant 1$ for all $\sigma \in S_{n}$. Suppose that the measure defining multiplicative 
function $d(\sigma)$ is such that $0<d^{-} \leqslant \hat{d}(j) \leqslant d^{+}$. Then we have

$$
\begin{aligned}
\Delta_{n}:=\left|M_{n}^{d}(f)-\exp \left\{\sum_{j=1}^{n} \hat{d}(j) \frac{\hat{f}(j)-1}{j}\right\}\right| \\
\leqslant c_{1}\left(\left(\sum_{j=0}^{n} p_{j}\right) \sum_{k=1}^{n}|\hat{f}(k)-1| p_{n-k}+\frac{1}{n^{d^{-}}} \sum_{k=1}^{n}|\hat{f}(k)-1| k^{d^{-}-1}\right. \\
\left.\quad+\frac{1}{n} \sum_{k=1}^{n}|\hat{f}(k)-1|\right)
\end{aligned}
$$

for $d^{-}<1$ and

$$
\Delta_{n} \leqslant c_{1}\left(\left(\sum_{j=0}^{n} p_{j}\right)^{-1} \sum_{k=1}^{n}|\hat{f}(k)-1| p_{n-k}+\frac{1}{n} \sum_{k=1}^{n}|\hat{f}(k)-1|\left(1+\log \frac{n}{k}\right)\right)
$$

for $d^{-} \geqslant 1$, where $c_{1}=c_{1}\left(d^{-}, d^{+}\right)$is a positive constant which depends on $d^{-}$ and $d^{+}$only, and

$$
p_{n}=\frac{1}{n !} \sum_{\sigma \in S_{n}} d(\sigma)=\left[z^{n}\right] \exp \left\{\sum_{j=1}^{\infty} \frac{\hat{d}(j)}{j} z^{j}\right\}
$$

Thus Theorem 3 shows that condition (11) in Theorem A is superfluous. The inequality of our theorem also yields more accurate estimate of the remainder term than Theorem $\mathrm{A}$.

Note that if function $h(\sigma)$ is additive then function $\exp (i t h(\sigma))$ is multiplicative which means that the characteristic function of an additive function with respect to our measure (10) is a mean value of a multiplicative function. It follows hence that the estimates for the mean values of multiplicative functions allow us to obtain information on the distribution of the values of additive functions.

Let us denote

$$
A(n)=\sum_{k=1}^{n} \hat{d}(k) \frac{\hat{h}_{n}(k)}{k}, \quad C_{n}=\sum_{j=1}^{n} \hat{d}(j) \frac{\hat{h}_{n}(j)}{j}\left(\frac{p_{n-j}}{p_{n}}-1\right),
$$

and

$$
L_{n, p}=\sum_{k=1}^{n} \frac{\left|\hat{h}_{n}(k)\right|^{p}}{k}, \quad L_{n, 2}^{\prime}=\sum_{j=1}^{n} \frac{\hat{h}_{n}^{2}(j)}{j}\left|\frac{p_{n-j}}{p_{n}}-1\right| .
$$

Henceforth we assume that $\tilde{h}_{n}(k)$ satisfies the normalizing condition

$$
\sum_{k=1}^{n} \hat{d}(k) \frac{\hat{h}_{n}^{2}(k)}{k}=1
$$


Theorem 4. Suppose $0<d^{-} \leqslant d_{j} \leqslant d^{+}$, and $p$ is a fixed number such that $\infty \geqslant p>\max \left\{2,1 / d^{-}\right\}$. Suppose

$$
F_{n}(x)=\nu_{n, d}(h(\sigma)-A(n)<x),
$$

where $h(\sigma)$ is a additive function satisfying the normalizing condition (12). Then we have

$$
\sup _{x \in \mathbb{R}}\left|F_{n}(x)-\Phi(x)+\frac{1}{\sqrt{2 \pi}} e^{-x^{2} / 2} C_{n}\right| \ll L_{n, 3}+L_{n, p}^{2 / p}+L_{n, 2}^{\prime},
$$

here we assume that

$$
L_{n, \infty}^{1 / \infty}=\lim _{p \rightarrow \infty} L_{n, p}^{1 / p}=\max _{1 \leqslant j \leqslant n}|\hat{h}(j)|
$$

for $p=\infty$.

Theorem 4 4 generalizes the corresponding result of Manstavičius [8] that was proved for the case of uniform measures $\hat{d}(j) \equiv 1$, later generalized for Ewens measures $\hat{d}(j) \equiv \theta>0$ in our paper [15].

\section{Proofs}

\subsection{Voronoi summation method}

Throughout the proofs we will routinely use a simple inequality

$$
b_{0}+b_{1}+\cdots+b_{n} \leqslant \sum_{k=0}^{n} b_{k} e^{1-k / n} \leqslant \sum_{k=0}^{\infty} b_{k} e^{1-k / n}=e b\left(e^{-1 / n}\right)
$$

for partial sums of coefficients of a generating function $b(x)=\sum_{k=0}^{\infty} b_{k} x^{k}$ with nonnegative coefficients $b_{k} \geqslant 0$. The next theorem shows that a similar lower bound is also valid if the logarithmic derivative of the generating function $b(x)$ does not grow too fast as $x \rightarrow 1$.

Theorem 5. Let $b(x)=\sum_{k=0}^{\infty} b_{k} x^{k}$ be a series with non-negative $b_{k} \geqslant 0$ coefficients, that converges in the interval $x \in[0,1)$. Suppose there exists such $c>0$ that the logarithmic derivative of $b(x)$ satisfies the inequality

$$
\frac{b^{\prime}(x)}{b(x)} \leqslant \frac{c}{1-x}
$$

for all $0 \leqslant x<1$. Then there exists such a positive constant $K=K(c)$ that

$$
\sum_{k=0}^{N} b_{k} \geqslant K(c) b\left(e^{-1 / N}\right)
$$

for all $N \geqslant 2 c$. 
Proof. Suppose $0 \leqslant x<1$ and $N \geqslant 2 c$, then

$$
\begin{aligned}
b(x) & \leqslant \sum_{k=0}^{N} b_{k} x^{k}+\frac{1}{N} \sum_{k=0}^{\infty} k b_{k} x^{k} \leqslant \sum_{k=0}^{N} b_{k}+\frac{x b^{\prime}(x)}{N} \\
& \leqslant \sum_{k=0}^{N} b_{k}+b(x) \frac{x}{N} \frac{b^{\prime}(x)}{b(x)} \leqslant \sum_{k=0}^{N} b_{k}+b(x) \frac{c x}{N(1-x)},
\end{aligned}
$$

here we have applied the inequality (14) satisfied by the logarithmic derivative of $b(x)$. Inserting into the above inequality $x=e^{-2 c / N}$ and estimating $\frac{e^{-2 c / N}}{1-e^{-2 c / N}} \leqslant \frac{N}{2 c}$ we obtain

$$
b\left(e^{-2 c / N}\right) \leqslant \sum_{k=0}^{N} b_{k}+\frac{1}{2} b\left(e^{-2 c / N}\right),
$$

which leads to the inequality

$$
\frac{1}{2} b\left(e^{-2 c / N}\right) \leqslant \sum_{k=0}^{N} b_{k} .
$$

If $c \leqslant 1 / 2$, then $e^{-1 / N} \leqslant e^{-2 c / N}$ therefore

$$
\frac{1}{2} b\left(e^{-1 / N}\right) \leqslant \frac{1}{2} b\left(e^{-2 c / N}\right) \leqslant \sum_{k=0}^{N} b_{k} .
$$

This means that for $c \leqslant 1 / 2$ the theorem will be true with $K(c)=\frac{1}{2}$.

Suppose now that $c>\frac{1}{2}$, then $e^{-1 / N} \geqslant e^{-2 c / N}$. Let us show that the ratio $b\left(e^{-2 c / N}\right) / b\left(e^{-1 / N}\right)$ is bounded from bellow. Using the upper bound (14) for the logarithmic derivative of $b(x)$ we get

$$
\begin{aligned}
\frac{b\left(e^{-2 c / N}\right)}{b\left(e^{-1 / N}\right)} & =\exp \left\{\log b\left(e^{-2 c / N}\right)-\log b\left(e^{-1 / N}\right)\right\}=\exp \left\{-\int_{e^{-2 c / N}}^{e^{-1 / N}} \frac{b^{\prime}(x)}{b(x)} d x\right\} \\
& \geqslant \exp \left\{-c \int_{e^{-2 c / N}}^{e^{-1 / N}} \frac{d x}{1-x}\right\}=\left(\frac{1-e^{-1 / N}}{1-e^{-2 c / N}}\right)^{c} \\
& \geqslant \frac{e^{-c / N}}{(2 c)^{c}} \geqslant \frac{e^{-1 / 2}}{(2 c)^{c}},
\end{aligned}
$$

since $N \geqslant 2 c$. This estimate together with inequality (15) proves that the statement of the theorem is true with $K(c)=\frac{e^{-1 / 2}}{2(2 c)^{c}}$, when $c>1 / 2$.

The theorem is proved. 
Throughout this section $p(z)$ will be defined as

$$
p(z)=\exp \left\{\sum_{k=1}^{\infty} \frac{d_{k}}{k} z^{k}\right\}=\sum_{j=0}^{\infty} p_{j} z^{j} .
$$

We will assume that $d_{k}$ are bounded from above and below by some fixed positive constants $0<d^{-} \leqslant d_{k} \leqslant d^{+}$, and denote $\theta:=\min \left\{1, d^{-}\right\}$. We will also denote $\tilde{d}_{k}=d_{k}-\theta$ and

$$
\tilde{p}(z)=\exp \left\{\sum_{k=1}^{\infty} \frac{\tilde{d}_{k}}{k} z^{k}\right\}=\sum_{n=0}^{\infty} \tilde{p}_{n} z^{n} .
$$

The relationship $\tilde{d}_{k}=d_{k}-\theta$ immediately leads to the identity for the corresponding generating functions

$$
\tilde{p}(z)=\exp \left\{\sum_{j=1}^{\infty} \frac{d_{j}-\theta}{j} z^{j}\right\}=p(z)(1-z)^{\theta} .
$$

In order to prove the inequality of our main Theorem 1 we will need some estimates for the asymptotic behavior of the coefficients of $p_{j}$ and $\tilde{p}_{j}$ of $p(z)$.

Differentiating $p(z)$ and $\tilde{p}(z)$ we conclude that these functions satisfy differential equations

$$
z p^{\prime}(z)=p(z) \sum_{k=1}^{\infty} d_{k} z^{k} \quad \text { and } \quad z \tilde{p}^{\prime}(z)=\tilde{p}(z) \sum_{k=1}^{\infty} \tilde{d}_{k} z^{k},
$$

which lead to the recurrent relationships for the coefficients $p_{n}$ and $\tilde{p}_{n}$ in the Taylor expansions of the corresponding functions

$$
p_{n}=\frac{1}{n} \sum_{k=1}^{n} d_{k} p_{n-k} \quad \text { and } \quad \tilde{p}_{n}=\frac{1}{n} \sum_{k=1}^{n} \tilde{d}_{k} \tilde{p}_{n-k},
$$

for $n \geqslant 1$. Taking the maximum of $d_{k}$ on the right hand side of the above equations and using the inequality (13) for partial sums of $p_{j}$ and $\tilde{p}_{j}$ we obtain the inequalities

$$
p_{n} \leqslant d^{+} e \frac{p\left(e^{-1 / n}\right)}{n} \quad \text { and } \quad \tilde{p}_{n} \leqslant d^{+} e \frac{\tilde{p}\left(e^{-1 / n}\right)}{n}
$$

that provide an upper bound for the coefficients $p_{n}$ and $\tilde{p}_{n}$.

A similar lower bound for $p_{n}$ has been proven in [10], stating that there is a positive constant $K\left(d^{+}\right)$such that

$$
p_{n} \geqslant d^{-} K\left(d^{+}\right) \frac{p\left(e^{-1 / n}\right)}{n} .
$$


An independent proof of this estimate can be based on the inequality of Theorem 5. Indeed, for $b(z)=p(z)$ we see that the condition of Theorem 5 is satisfied with $c=d^{+}$as $\frac{p^{\prime}(x)}{p(x)}=\sum_{j=1}^{\infty} d_{j} x^{j-1} \leqslant \frac{d^{+}}{1-x}$, for $0<x<1$. This gives us the lower bound for partial sums of $p_{j}$, which together with recurrent relationships (16) satisfied by $p_{n}$ yields the proof of the lower bound (18) for $p_{n}$.

Lemma 1. If $m \geqslant n \geqslant 1$, then

$$
\left(\frac{m}{n}\right)^{d^{-}} e^{-d^{-} / n} \leqslant \frac{p\left(e^{-1 / m}\right)}{p\left(e^{-1 / n}\right)} \leqslant\left(\frac{m}{n}\right)^{d^{+}} e^{d^{+} / m}
$$

and

$$
\left(\frac{m}{n}\right)^{\tilde{d}^{-}} e^{-\tilde{d}^{-} / n} \leqslant \frac{\tilde{p}\left(e^{-1 / m}\right)}{\tilde{p}\left(e^{-1 / n}\right)} \leqslant\left(\frac{m}{n}\right)^{\tilde{d}^{+}} e^{\tilde{d}^{+} / m}
$$

where $\tilde{d}_{k}^{+}=d_{k}^{+}-\theta, \tilde{d}_{k}^{-}=d_{k}^{-}-\theta$ and $\tilde{d}^{+}=d^{+}-\theta$.

Proof. We have

$$
\begin{aligned}
\frac{p\left(e^{-1 / m}\right)}{p\left(e^{-1 / n}\right)} & =\exp \left\{\sum_{k=1}^{\infty} \frac{d_{k}}{k}\left(e^{-k / m}-e^{-k / n}\right)\right\} \leqslant \exp \left\{d^{+} \sum_{k=1}^{\infty} \frac{e^{-k / m}-e^{-k / n}}{k}\right\} \\
& =\exp \left\{d^{+} \log \frac{1-e^{-1 / n}}{1-e^{-1 / m}}\right\}=\left(\frac{1-e^{-1 / n}}{1-e^{-1 / m}}\right)^{d^{+}} \leqslant\left(\frac{m}{n}\right)^{d^{+}} e^{d^{+} / m}
\end{aligned}
$$

here we have used the inequalities $e^{-x} x \leqslant 1-e^{-x} \leqslant x$ for $x \geqslant 0$.

In the same way we obtain the lower bound estimate.

The proof of the second inequality is analogous.

The next lemma proves that sequence $p_{n}$ varies "smoothly" in a certain sense.

Lemma 2. If $0 \leqslant s \leqslant n / 2$, then

$$
\left|p_{n+s}-p_{n}\right| \ll p_{n}\left(\frac{s}{n}\right)^{\theta}
$$

where $\theta=\min \left\{d^{-}, 1\right\}$.

Proof. Generating function $p(z)$ can be represented as a product $p(z)=\frac{\tilde{p}(z)}{(1-z)^{\theta}}$. This allow us to express the coefficients of $p(z)$ as a convolution of coefficients of the corresponding generating functions

$$
p_{n}=\sum_{k=0}^{n} \tilde{p}_{k}\left(\begin{array}{c}
n-k+\theta-1 \\
n-k
\end{array}\right) .
$$


The idea behind the proof is to exploit the fact that binomial coefficients $\left(\begin{array}{c}n-k+\theta-1 \\ n-k\end{array}\right)$ occurring in the above expression vary smoothly as follows from the classical estimate

$$
\left(\begin{array}{c}
n+\theta-1 \\
n
\end{array}\right)=\frac{n^{\theta-1}}{\Gamma(\theta)}\left(1+O\left(\frac{1}{n}\right)\right)
$$

(see e. g. [3]). We can represent $p_{n+s}-p_{n}$ as a difference of convolutions

$$
\begin{aligned}
p_{n+s}-p_{n}= & \sum_{k=0}^{n} \tilde{p}_{k}\left(\left(\begin{array}{c}
n+s-k+\theta-1 \\
n+s-k
\end{array}\right)-\left(\begin{array}{c}
n-k+\theta-1 \\
n-k
\end{array}\right)\right) \\
& +\sum_{n+s \geqslant k>n} \tilde{p}_{k}\left(\begin{array}{c}
n+s-k+\theta-1 \\
n+s-k
\end{array}\right)=: S_{1}+S_{2} .
\end{aligned}
$$

If $s=0$, then the estimate of the theorem is trivial, therefore we assume that $s>0$. Applying here the upper bound (17) for $p_{j}$ together with estimates for ratio $\frac{\tilde{p}\left(e^{-1 /(n+s)}\right)}{\tilde{p}\left(e^{-1 / n}\right)}$ provided by Lemma 1 we obtain

$$
\begin{aligned}
S_{2} & \leqslant \max _{n+s \geqslant k>n} \tilde{p}_{k} \sum_{l=0}^{s}\left(\begin{array}{c}
l+\theta-1 \\
l
\end{array}\right) \leqslant \tilde{p}\left(e^{-1 / n}\right) e d^{+} \frac{\tilde{p}\left(e^{-1 /(n+s)}\right)}{\tilde{p}\left(e^{-1 / n}\right)} \frac{1}{n}\left(\begin{array}{c}
s+\theta \\
s
\end{array}\right) \\
& \ll s \frac{\tilde{p}\left(e^{-1 / n}\right)}{n}=s^{\theta} \frac{p\left(e^{-1 / n}\right)\left(1-e^{-1 / n}\right)^{\theta}}{n} \leqslant\left(\frac{s}{n}\right)^{\theta} \frac{p\left(e^{-1 / n}\right)}{n} .
\end{aligned}
$$

here while dealing with the binomial coefficient $\left(\begin{array}{c}s+\theta \\ s\end{array}\right)$ we used (19).

If $\theta=1$, then $S_{1}=0$, therefore estimating $S_{1}$ we may assume that $\theta<1$. First we split the sum $S_{1}$ into two parts and and notice that the binomial coefficients $\left(\begin{array}{c}l+\theta-1 \\ l\end{array}\right)$ for $0<\theta<1$ are monotonously decreasing when $l$ is increasing, which implies that $0<\left(\begin{array}{c}n-k+\theta-1 \\ n-k\end{array}\right)-\left(\begin{array}{c}n+s-k+\theta-1 \\ n+s-k\end{array}\right) \leqslant\left(\begin{array}{c}n-k+\theta-1 \\ n-k\end{array}\right)$. Hence splitting sum $S_{1}$ into two parts we obtain

$$
\begin{aligned}
\left|S_{1}\right| \leqslant & \sum_{k=0}^{n-s} \tilde{p}_{k}\left(\begin{array}{c}
n-k+\theta-1 \\
n-k
\end{array}\right)\left|\frac{\left(\begin{array}{c}
n+s-k+\theta-1 \\
n+s-k
\end{array}\right)}{\left(\begin{array}{c}
n-k+\theta-1 \\
n-k
\end{array}\right)}-1\right| \\
& +\sum_{n-s<k \leqslant n} \tilde{p}_{k}\left(\begin{array}{c}
n-k+\theta-1 \\
n-k
\end{array}\right)
\end{aligned}
$$

Once again applying Lemma 1, the upper bound (17) for $\tilde{p}_{j}$ and the asymptotic 
(19) for binomial coefficients, we obtain

$$
\begin{aligned}
S_{1} & \ll \sum_{k=0}^{n-s} \tilde{p}_{k}\left(\begin{array}{c}
n-k+\theta-1 \\
n-k
\end{array}\right) \frac{s}{n-k}+\frac{\tilde{p}\left(e^{-1 / n}\right)}{n} \sum_{0 \leqslant j<s}\left(\begin{array}{c}
j+\theta-1 \\
j
\end{array}\right) \\
& \ll \frac{s}{n} n^{\theta-1} \sum_{k \leqslant n / 2} \tilde{p}_{k}+\sum_{n / 2<k \leqslant n-s} \tilde{p}_{k} s(n-k)^{\theta-2}+\frac{p\left(e^{-1 / n}\right)}{n}\left(\frac{s}{n}\right)^{\theta} \\
& \ll \frac{s}{n} n^{\theta-1} \tilde{p}\left(e^{-1 / n}\right)+\frac{\tilde{p}\left(e^{-1 / n}\right)}{n} s \sum_{l \geqslant s} l^{\theta-2}+\frac{p\left(e^{-1 / n}\right)}{n}\left(\frac{s}{n}\right)^{\theta} \\
& \ll \frac{p\left(e^{-1 / n}\right)}{n} \frac{s}{n}+\frac{p\left(e^{-1 / n}\right)}{n}\left(\frac{s}{n}\right)^{\theta} \ll \frac{p\left(e^{-1 / n}\right)}{n}\left(\frac{s}{n}\right)^{\theta} .
\end{aligned}
$$

The lemma is proved.

For $0 \leqslant x \leqslant 1$ we denote

$$
G_{x}(z)=\frac{p(z)}{p(z x)}=\sum_{k=0}^{\infty} g_{k, x} z^{k} \quad \text { and } \quad \tilde{G}_{x}(z)=\frac{\tilde{p}(z)}{\tilde{p}(z x)}=\sum_{k=0}^{\infty} \tilde{g}_{k, x} z^{k},
$$

and

$$
C_{x}(z)=\left(\frac{1-z x}{1-z}\right)^{\theta}=\sum_{k=0}^{\infty} c_{k, x} z^{k} .
$$

Since $\tilde{p}(z)=p(z)(1-z)^{\theta}$, we have

$$
G_{x}(z)=\tilde{G}_{x}(z)\left(\frac{1-z x}{1-z}\right)^{\theta}
$$

Differentiating functions $C_{x}(z)$ and $G_{x}(z)$ with respect to $z$ we conclude that they satisfy differential equations

$$
z C_{x}^{\prime}(z)=C_{x}(z) \theta \sum_{k=1}^{\infty} z^{k}\left(1-x^{k}\right) \quad \text { and } \quad z G_{x}^{\prime}(z)=G_{x}(z) \sum_{k=1}^{\infty} d_{k} z^{k}\left(1-x^{k}\right) .
$$

which are equivalent to the recurrent relationships

$$
c_{n, x}=\frac{\theta}{n} \sum_{k=1}^{n} c_{n-k, x}\left(1-x^{k}\right) \quad \text { and } \quad g_{n, x}=\frac{1}{n} \sum_{k=1}^{n} g_{n-k, x} d_{k}\left(1-x^{k}\right),
$$

satisfied by the coefficients $c_{n, x}, g_{n, x}$ in the Taylor expansions of the corresponding functions, for all $n \geqslant 1$ with initial conditions $c_{0, x}=g_{0, x}=1$. Since the coefficients of these linear recurrences together with the initial conditions are 
non-negative, thus it follows by induction that the solutions $c_{n, x}, g_{n, x}$ of the above recurrences are also non-negative $c_{n, x}, g_{n, x} \geqslant 0$. Therefore we can apply our inequality (13) for the partial sums of coefficients of generating functions with non-negative coefficients to obtain the upper bounds

$$
\sum_{m=0}^{n} c_{m, x} \leqslant e C_{x}\left(e^{-1 / n}\right) \quad \text { and } \quad \sum_{m=0}^{n} g_{m, x} \leqslant e G_{x}\left(e^{-1 / n}\right) .
$$

Applying these upper bounds for partial sums of $c_{n, x}, g_{n, x}$ to bound the right hand side of the recurrences (20) satisfied by these coefficients we obtain the inequalities

$$
c_{n, x} \leqslant \frac{e \theta C_{x}\left(e^{-1 / n}\right)}{n} \quad \text { and } \quad g_{n, x} \leqslant \frac{e d^{+} G_{x}\left(e^{-1 / n}\right)}{n} .
$$

The same considerations applied to generating function $\tilde{G}_{x}(z)$ lead to inequality

$$
\tilde{g}_{n, x} \leqslant \frac{e\left(d^{+}-\theta\right) \tilde{G}_{x}\left(e^{-1 / n}\right)}{n} .
$$

Lemma 3. Suppose $0<x<1$ and $s \leqslant m / 2$, then we have

$$
\left|c_{m, x}-c_{m-s, x}\right| \ll s m^{\theta-2}(1-x)^{\theta}+\frac{s}{m^{2}},
$$

for $m \geqslant 1$.

Proof. We will apply the same standard technique of contour integration that was used by Flajolet and Odlyzko [3] to analyze generating functions with singularities of type $1 /(1-z)^{\alpha}$. The first step in our proof is to represent $c_{m, x}$ as a Cauchy integral of function $C_{x}(z)$ over contour $L$ that consists of four parts $L=L_{1} \cup L_{2} \cup$ $L_{3} \cup L_{4}$, two arcs $L_{1}, L_{2}$ with radiuses $2,1 / m$ correspondingly and two segments $L_{3}$ and $L_{4}$ connecting the ends of these arcs as shown bellow on the picture.

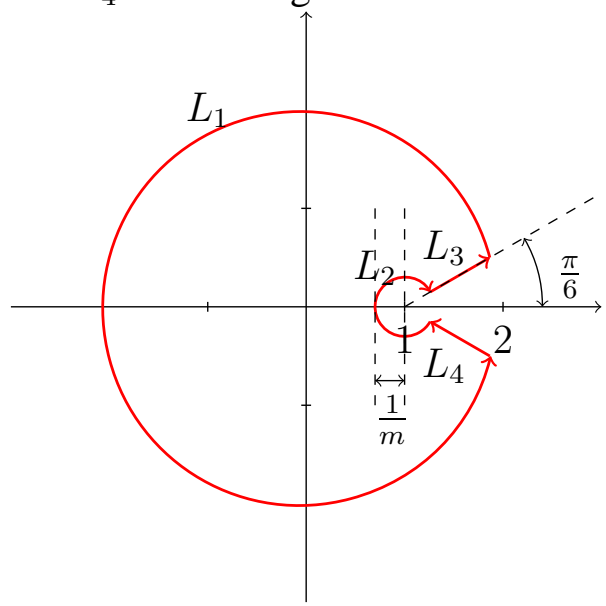


Thus we can replace the difference of coefficients $c_{m, x}$ and $c_{m-1, x}$ by the difference of the corresponding Cauchy integrals

$$
\begin{aligned}
\left|c_{m, x}-c_{m-1, x}\right| & =\left|\frac{1}{2 \pi i} \int_{L} C_{x}(z) \frac{(1-z)}{z^{m+1}} d z\right| \leqslant \frac{1}{2 \pi} \int_{L} \frac{|1-x z|^{\theta}|1-z|^{1-\theta}}{|z|^{m+1}}|d z| \\
& \leqslant \frac{1}{2 \pi} \int_{L} \frac{(|1-z|+|z||1-x|)^{\theta}|1-z|^{1-\theta}}{|z|^{m+1}}|d z| \\
& \ll \int_{L} \frac{|1-z|+|1-z|^{1-\theta}|1-x|^{\theta}}{|z|^{m+1}}|d z| .
\end{aligned}
$$

Let us now estimate the above integral over the four separate parts of our contour

$$
\begin{aligned}
& \left|c_{m, x}-c_{m-1, x}\right| \ll \frac{1}{2^{m}}+\int_{1+\frac{1}{m}}^{2} \frac{(y-1)+(y-1)^{1-\theta}|1-x|^{\theta}}{y^{m+1}} d y \\
& +\int_{|z-1|=\frac{1}{m}} \frac{|1-z|+|1-z|^{1-\theta}|1-x|^{\theta}}{|z|^{m+1}}|d z| \\
& \ll \frac{(1-x)^{\theta}}{m} \int_{m \log \left(1+\frac{1}{m}\right)}^{m \log 2} \frac{\left(e^{u / m}-1\right)^{1-\theta}}{e^{u}} d u+\frac{1}{m^{2}}+m^{\theta-2}(1-x)^{\theta} \\
& \ll(1-x)^{\theta} m^{\theta-2} \int_{m \log \left(1+\frac{1}{m}\right)}^{m \log 2} u^{\theta} e^{-u} d u+\frac{1}{m^{2}}+m^{\theta-2}(1-x)^{\theta} \\
& \ll m^{\theta-2}(1-x)^{\theta}+\frac{1}{m^{2}} \text {. }
\end{aligned}
$$

From the above estimate of the difference $\left|c_{m, x}-c_{m-1, x}\right|$ by the standard use of telescoping sums we obtain the estimate

$$
\begin{aligned}
\left|c_{m, x}-c_{m-s, x}\right| & \leqslant\left|c_{m, x}-c_{m-1, x}\right|+\left|c_{m-1, x}-c_{m-2, x}\right|+\cdots+\left|c_{m-s+1, x}-c_{m-s, x}\right| \\
& \ll s m^{\theta-2}(1-x)^{\theta}+\frac{s}{m^{2}},
\end{aligned}
$$

for $s \leqslant m / 2$.

The lemma is proved.

Lemma 4. For $0 \leqslant x \leqslant e^{-1 / n}$ and $k \leqslant n / 8$, we have

$$
g_{n, x}-g_{n-k, x} \ll \frac{p\left(e^{-1 / n}\right)}{n p(x)}\left(\left(\frac{k}{n}\right)^{\theta}+\frac{1}{(n(1-x))^{\theta}}\right) .
$$

Proof. Since $G_{x}(z)=\tilde{G}_{x}(z) C_{x}(z)$ this allows us to express the difference $g_{n, x}$ as a convolution of coefficients of $\tilde{G}_{x}(z)$ and $C_{x}(z)$. Thus we can to express the 
difference of coefficients $g_{n, x}$ as

$$
\begin{aligned}
g_{n, x}-g_{n-k, x}= & \sum_{s=0}^{n} \tilde{g}_{s, x} c_{n-s, x}-\sum_{s=0}^{n-k} \tilde{g}_{s, x} c_{n-k-s, x}=\sum_{s=0}^{n-2 k} \tilde{g}_{s, x}\left(c_{n-s, x}-c_{n-k-s, x}\right) \\
& +\sum_{n-2 k<s \leqslant n} \tilde{g}_{s, x} c_{n-s, x}-\sum_{n-2 k<s \leqslant n-k} \tilde{g}_{s, x} c_{n-k-s, x}=: S_{1}+S_{2}+S_{3} .
\end{aligned}
$$

If $\theta=1$ then all $c_{j, x}$ with $j \geqslant 1$ are equal $c_{j, x}=1-x$. Which means that $S_{1}=0$ if $\theta=1$. Therefore, while estimating $S_{1}$, we may assume that $\theta<1$. Applying the estimate of the difference $c_{j+s, x}-c_{j, x}$ of Lemma 3 together with the inequality (13) for partial sums of coefficients of generating function with positive coefficients and making use of the upper bound (22) for $\tilde{g}_{j, x}$ we obtain

$$
\begin{aligned}
S_{1} \ll & \sum_{0 \leqslant s \leqslant n-2 k} \tilde{g}_{s, x}\left(k(n-s)^{\theta-2}(1-x)^{\theta}+\frac{k}{(n-s)^{2}}\right) \\
\ll & k n^{\theta-2}(1-x)^{\theta} \sum_{0 \leqslant s \leqslant n / 2} \tilde{g}_{s, x} \\
& +\sum_{n / 2 \leqslant s \leqslant n-2 k} \tilde{g}_{s, x}\left(k(n-s)^{\theta-2}(1-x)^{\theta}+\frac{k}{(n-s)^{2}}\right) \\
\ll & k n^{\theta-2}(1-x)^{\theta} \tilde{G}_{x}\left(e^{-1 / n}\right) \\
& +k \frac{\tilde{G}_{x}\left(e^{-1 / n}\right)}{n} \sum_{n / 2 \leqslant s \leqslant n-2 k}\left((n-s)^{\theta-2}(1-x)^{\theta}+\frac{1}{(n-s)^{2}}\right) \\
& \ll k n^{\theta-2}(1-x)^{\theta} G_{x}\left(e^{-1 / n}\right)\left(\frac{1-e^{-1 / n}}{1-x e^{-1 / n}}\right)^{\theta} \\
& +k \frac{G_{x}\left(e^{-1 / n}\right)}{n}\left(\frac{1-e^{-1 / n}}{1-x e^{-1 / n}}\right)^{\theta}\left(k^{\theta-1}(1-x)^{\theta}+\frac{1}{k}\right) .
\end{aligned}
$$

Since $1-x e^{-1 / n} \geqslant 1-x$, we have

$$
S_{1} \ll \frac{k}{n^{2}} \frac{p\left(e^{-1 / n}\right)}{p\left(x e^{-1 / n}\right)}+\frac{1}{n} \frac{p\left(e^{-1 / n}\right)}{p\left(x e^{-1 / n}\right)}\left(\left(\frac{k}{n}\right)^{\theta}+\frac{1}{(n(1-x))^{\theta}}\right) .
$$


In a similar way we obtain

$$
\begin{aligned}
S_{2}+S_{3} & \ll \frac{1}{n} \tilde{G}_{x}\left(e^{-1 / n}\right) \sum_{0 \leqslant l \leqslant 2 k} c_{x, l} \\
& \ll \frac{1}{n} \frac{p\left(e^{-1 / n}\right)}{p\left(x e^{-1 / n}\right)}\left(\frac{1-e^{-1 / n}}{1-x e^{-1 / n}}\right)^{\theta}\left(\frac{1-x e^{-1 / 2 k}}{1-e^{-1 / 2 k}}\right)^{\theta} \\
& \ll \frac{1}{n} \frac{p\left(e^{-1 / n}\right)}{p\left(x e^{-1 / n}\right)}\left(\frac{1-x+x\left(1-e^{-1 / 2 k}\right)}{1-x}\right)^{\theta}\left(\frac{k}{n}\right)^{\theta} \\
& \ll \frac{1}{n} \frac{p\left(e^{-1 / n}\right)}{p\left(x e^{-1 / n}\right)}\left(1+\frac{1}{k(1-x)}\right)^{\theta}\left(\frac{k}{n}\right)^{\theta} \\
& \ll \frac{1}{n} \frac{p\left(e^{-1 / n}\right)}{p\left(x e^{-1 / n}\right)}\left(\left(\frac{k}{n}\right)^{\theta}+\frac{1}{(n(1-x))^{\theta}}\right) .
\end{aligned}
$$

Since $p\left(x e^{-1 / n}\right) \gg p(x)$ if $0 \leqslant x \leqslant e^{-1 / n}$, the proof of the lemma follows.

Lemma 5. Suppose $u(x)=\exp \left\{\sum_{k=1}^{\infty} \frac{u_{k}}{k} x^{k}\right\}$ and $0 \leqslant u_{k} \leqslant A$, then the following estimates hold:

1)

$$
\int_{0}^{1} \frac{x^{j-1}}{u(x)} d x \ll \frac{1}{j u\left(e^{-1 / j}\right)} \quad \text { if } \quad j \geqslant 1
$$

2)

$$
\int_{0}^{e^{-1 / n}} \frac{x^{j-1}}{u(x)} d x \ll \frac{e^{-j / n}}{j u\left(e^{-1 / n}\right)} \quad \text { if } \quad j \geqslant n \geqslant 1 .
$$

Proof. 1) For $j \geqslant 1$ we can split the integral into two parts and taking into account that $u(x)$ is increasing obtain

$$
\begin{aligned}
\int_{0}^{1} \frac{x^{j-1}}{u(x)} d x & =\int_{0}^{e^{-1 / j}} \frac{x^{j-1}}{u(x)} d x+\int_{e^{-1 / j}}^{1} \frac{x^{j-1}}{u(x)} d x \\
& \leqslant \frac{1}{u\left(e^{-1 / j}\right)} \int_{0}^{e^{-1 / j}} \frac{u\left(e^{-1 / j}\right)}{u(x)} x^{j-1} d x+\frac{1-e^{-1 / j}}{j u\left(e^{-1 / j}\right)} \\
& \leqslant \frac{1}{u\left(e^{-1 / j}\right)} \int_{0}^{e^{-1 / j}} x^{j-1} \exp \left\{\sum_{k=1}^{\infty} \frac{u_{k}}{k}\left(e^{-k / j}-x^{k}\right)\right\} d x+\frac{1}{j u\left(e^{-1 / j}\right)}
\end{aligned}
$$

Applying now the upper bound $u_{k} \leqslant A$ to estimate the quantity under the integra- 
tion sign we obtain

$$
\begin{aligned}
\int_{0}^{1} \frac{x^{j-1}}{u(x)} d x & \leqslant \frac{1}{u\left(e^{-1 / j}\right)} \int_{0}^{e^{-1 / j}} x^{j-1}\left(\frac{1-x}{1-e^{-1 / j}}\right)^{A} d x+\frac{1}{j u\left(e^{-1 / j}\right)} \\
& \leqslant \frac{e^{A / j} j^{A}}{u\left(e^{-1 / j}\right)} \int_{0}^{e^{-1 / j}} x^{j-1}(1-x)^{A} d x+\frac{1}{j u\left(e^{-1 / j}\right)} \\
& =\frac{e^{A / j} j^{A}}{u\left(e^{-1 / j}\right)} \int_{1 / j}^{\infty}\left(1-e^{-y}\right)^{A} e^{-j y} d y+\frac{1}{j u\left(e^{-1 / j}\right)} \\
& \leqslant \frac{e^{A / j} j^{A}}{u\left(e^{-1 / j}\right)} \int_{0}^{\infty} y^{A} e^{-j y} d y+\frac{1}{j u\left(e^{-1 / j}\right)}=\frac{e^{A / j} \Gamma(A+1)+1}{j u\left(e^{-1 / j}\right)}
\end{aligned}
$$

here we have used the inequalities $e^{-y} y \leqslant 1-e^{-y} \leqslant y$, for $y \geqslant 0$.

2) Suppose now that $j \geqslant n$. Applying the same considerations that we used to estimate the integral over region $0 \leqslant x \leqslant e^{-1 / n}$ in the previous estimate, we obtain

$$
\begin{aligned}
\int_{0}^{e^{-1 / n}} \frac{x^{j-1}}{u(x)} d x & \leqslant \frac{1}{u\left(e^{-1 / n}\right)} \int_{0}^{e^{-1 / n}} x^{j-1}\left(\frac{1-x}{1-e^{-1 / n}}\right)^{A} d x \\
& \leqslant \frac{e^{A / n} n^{A}}{u\left(e^{-1 / n}\right)} \int_{0}^{e^{-1 / n}} x^{j-1}(1-x)^{A} d x \\
& =\frac{e^{A / n} n^{A}}{u\left(e^{-1 / n}\right)} \int_{1 / n}^{\infty}\left(1-e^{-y}\right)^{A} e^{-j y} d y \\
& \leqslant \frac{e^{A / n} n^{A}}{u\left(e^{-1 / n}\right)} \int_{1 / n}^{\infty} y^{A} e^{-j y} d y \\
& =\frac{e^{A / n} n^{A}}{u\left(e^{-1 / n}\right)} \frac{1}{j^{A+1}} \int_{j / n}^{\infty} y^{A} e^{-y} d y \ll \frac{e^{-j / n}}{j u\left(e^{-1 / n}\right)}
\end{aligned}
$$

The last inequality follows from the fact that $\int_{T}^{\infty} y^{A} e^{-y} d y \ll T^{A} e^{-T}$, as $T \rightarrow \infty$.

The lemma is proved.

\section{Lemma 6.}

$$
\int_{0}^{e^{-1 / n}}\left|g_{x, n}-\frac{p_{n}}{p(x)}\right| x^{j-1} d x \ll \frac{p\left(e^{-1 / n}\right)}{n}\left(\frac{j^{\theta-1}}{n^{\theta}}\right) \frac{1}{p\left(e^{-1 / j}\right)},
$$

when $1 \leqslant j \leqslant n$.

Proof. We can represent function $p(z)$ as a product $p(z)=p(x z) \frac{p(z)}{p(x z)}=p(x z) G_{x}(z)$ which is equivalent to representation of the coefficient $p_{n}$ of $p(z)$ as a convolution 
$p_{n}=\sum_{k=0}^{n} p_{k} x^{k} g_{n-k, x}$. Applying this identity we obtain

$$
\begin{aligned}
\left|g_{x, n}-\frac{p_{n}}{p(x)}\right|= & \frac{1}{p(x)}\left|p(x) g_{n, x}-\sum_{k=0}^{n} p_{k} x^{k} g_{n-k, x}\right| \\
\leqslant & \frac{1}{p(x)} \sum_{k \leqslant n / 8} p_{k} x^{k}\left|g_{n, x}-g_{n-k, x}\right|+\frac{g_{n, x}}{p(x)} \sum_{k>n / 8} p_{k} x^{k} \\
& +\frac{1}{p(x)} \sum_{n / 8<k \leqslant n} p_{k} x^{k} g_{n-k, x},
\end{aligned}
$$

Suppose $0 \leqslant x \leqslant e^{-1 / n}$. Applying here Lemma 4 and the upper bound (17) for $p_{n}$ we have

$$
\begin{aligned}
\left|g_{x, n}-\frac{p_{n}}{p(x)}\right| \ll & \frac{p\left(e^{-1 / n}\right)}{n p(x)^{2}} \sum_{k \leqslant n / 8} p_{k} x^{k}\left(\left(\frac{k}{n}\right)^{\theta}+\frac{1}{(n(1-x))^{\theta}}\right) \\
& +\frac{G_{x}\left(e^{-1 / n}\right)}{n p(x)} \sum_{k>n / 8} p_{k} x^{k}+\frac{p\left(e^{-1 / n}\right)}{n} \frac{x^{n / 8}}{p(x)} G_{x}\left(e^{-1 / n}\right) \\
= & : S_{1}(x)+S_{2}(x)+S_{3}(x) .
\end{aligned}
$$

Applying Lemma $\left[\right.$ with $u(x)=p(x)^{2}$ and $u(x)=p(x)^{2}(1-x)^{\theta}$, for $1 \leqslant j \leqslant n$ we have

$$
\begin{aligned}
& \int_{0}^{e^{-1 / n}} S_{1}(x) x^{j-1} d x \\
& =\frac{p\left(e^{-1 / n}\right)}{n} \sum_{k \leqslant n / 8} p_{k}\left(\left(\frac{k}{n}\right)^{\theta} \int_{0}^{e^{-1 / n}} \frac{x^{k+j-1}}{p(x)^{2}} d x+\frac{1}{n^{\theta}} \int_{0}^{e^{-1 / n}} \frac{x^{k+j-1} d x}{p(x)^{2}(1-x)^{\theta}}\right) \\
& \ll \frac{p\left(e^{-1 / n}\right)}{n} \sum_{k \leqslant n / 8} p_{k}\left(\left(\frac{k}{n}\right)^{\theta} \frac{1}{(k+j) p\left(e^{-1 /(k+j))^{2}}\right.}\right. \\
& \ll \frac{p\left(e^{-1 / n}\right)}{n} \sum_{k \leqslant n / 8} \frac{1}{(k+j) p\left(e^{-1 /(k+j)}\right)^{2}}\left(\left(\frac{k}{n}\right)^{\theta}+\frac{(k+j)^{\theta}}{n^{\theta}}\right) \\
& \ll \frac{p\left(e^{-1 / n}\right)}{n}\left(\frac{1}{j}\left(\frac{j}{n}\right)^{\theta} \frac{1}{p\left(e^{-1 / j}\right)^{2}} \sum_{k \leqslant j} p_{k}+\sum_{k>j} \frac{p_{k}}{k}\left(\frac{k}{n}\right)^{\theta} \frac{1}{p\left(e^{-1 / k}\right)^{2}}\right) .
\end{aligned}
$$


Applying here inequality $\sum_{k \leqslant j} p_{k} \leqslant e p\left(e^{-1 / j}\right)$ in the first sum and the estimate (17) in the second one we have

$$
\begin{aligned}
& \int_{0}^{e^{-1 / n}} S_{1}(x) x^{j-1} d x \\
& \ll \frac{p\left(e^{-1 / n}\right)}{n}\left(\frac{1}{j}\left(\frac{j}{n}\right)^{\theta} \frac{1}{p\left(e^{-1 / j}\right)}+\frac{1}{p\left(e^{-1 / j}\right)} \sum_{k>j} \frac{1}{k^{2}}\left(\frac{k}{n}\right)^{\theta} \frac{p\left(e^{-1 / j}\right)}{p\left(e^{-1 / k}\right)}\right) .
\end{aligned}
$$

From the estimate $\frac{p\left(e^{-1 / j}\right)}{p\left(e^{-1 / k}\right)} \leqslant\left(\frac{j}{k}\right)^{d^{-}} e^{d^{-} / j}$ of Lemma 1 we obtain

$$
\begin{aligned}
& \int_{0}^{e^{-1 / n}} S_{1}(x) x^{j-1} d x \\
& \ll \frac{p\left(e^{-1 / n}\right)}{n}\left(\frac{1}{j}\left(\frac{j}{n}\right)^{\theta} \frac{1}{p\left(e^{-1 / j}\right)}+\frac{1}{p\left(e^{-1 / j}\right)} \sum_{k>j} \frac{1}{k^{2}}\left(\frac{k}{n}\right)^{\theta}\left(\frac{j}{k}\right)^{d^{-}}\right) \\
& \ll \frac{p\left(e^{-1 / n}\right)}{n}\left(\frac{j^{\theta-1}}{n^{\theta}}\right) \frac{1}{p\left(e^{-1 / j}\right)} .
\end{aligned}
$$

Let us now estimate $S_{2}(x)$

$$
S_{2}(x) \ll \frac{p\left(e^{-1 / n}\right)}{n p(x)^{2}} \sum_{k>n / 8} p_{k} x^{k} \leqslant \frac{p\left(e^{-1 / n}\right) x^{n / 16}}{n p(x)^{2}} p(\sqrt{x}) \ll \frac{p\left(e^{-1 / n}\right) x^{n / 16}}{n p(x)}
$$

since $p(y) \ll p\left(y^{2}\right)$ uniformly for $0 \leqslant y<1$. Hence, applying Lemma 5 we have

$$
\int_{0}^{e^{-1 / n}} S_{2}(x) x^{j-1} d x \ll \frac{p\left(e^{-1 / n}\right)}{n} \int_{0}^{e^{-1 / n}} \frac{x^{n / 16}}{p(x)} d x \ll \frac{1}{n^{2}} .
$$

In a similar way we obtain the estimate

$$
\int_{0}^{e^{-1 / n}} S_{3}(x) x^{j-1} d x \ll \frac{p\left(e^{-1 / n}\right)^{2}}{n} \int_{0}^{e^{-1 / n}} \frac{x^{n / 8}}{p(x)^{2}} d x \ll \frac{1}{n^{2}} .
$$

Collecting the obtained estimates and noticing that

$$
\frac{p\left(e^{-1 / n}\right)}{n}\left(\frac{j^{\theta-1}}{n^{\theta}}\right) \frac{1}{p\left(e^{-1 / j}\right)} \gg \frac{1}{n j} \geqslant \frac{1}{n^{2}},
$$

for $1 \leqslant j \leqslant n$, we obtain the proof of the lemma. 
Let us define

$$
V_{j}(z)=p(z) \int_{0}^{1} \frac{x^{j-1}}{p(z x)} d x=\sum_{m=0}^{\infty} v_{m, j} z^{m}
$$

Denoting

$$
q(z)=\frac{1}{p(z)}=\sum_{m=0}^{\infty} q_{m} z^{m}
$$

we can represent $V_{j}(z)$ as a product of two Taylor series

$$
V_{j}(z)=\sum_{m=0}^{\infty} v_{m, j} z^{m}=p(z) \int_{0}^{1} x^{j-1} q(z x) d x=\sum_{m=0}^{\infty} p_{m} z^{m} \sum_{s=0}^{\infty} \frac{q_{s}}{s+j} z^{s},
$$

which leads to representations of the Taylor coefficients of $V_{j}(z)$ as a convolution of the coefficients of the appropriate series

$$
v_{m, j}=\sum_{s=0}^{m} \frac{p_{m-s} q_{s}}{s+j}
$$

On the other hand,

$$
V_{j}(z)=\int_{0}^{1} x^{j-1} G_{x}(z) d x=\sum_{m=0}^{\infty} z^{m} \int_{0}^{1} g_{m, x} x^{j-1} d x=\sum_{m=0}^{\infty} v_{m, j} z^{m},
$$

which means that

$$
v_{m, j}=\int_{0}^{1} g_{m, x} x^{j-1} d x \geqslant 0
$$

Lemma 7. We have

$$
v_{m, j} \ll \frac{1}{j^{2}} \quad \text { when } \quad 1 \leqslant m \leqslant j
$$

and $v_{0, j}=\frac{1}{j}$.

Proof. Differentiating $V_{j}(z)$ we can easily check that this function satisfies differential equation

$$
z V_{j}^{\prime}(z)=V_{j}(z) \sum_{k=1}^{\infty} d_{k} z^{k}+1-j V_{j}(z) .
$$

Putting here $z=0$, we obtain $V_{j}(0)=v_{0, j}=1 / j$. 
Suppose that $m \geqslant 1$. The above differential equation for $V_{j}(z)$ is equivalent to the recurrence relation between the Taylor coefficients $v_{m, j}$, applying which we obtain

$$
\begin{aligned}
v_{m, j} & =\frac{1}{m+j} \sum_{k=1}^{m} d_{k} v_{m-k, j} \leqslant \frac{d^{+} e V_{j}\left(e^{-1 / m}\right)}{m+j}=\frac{d^{+} e}{m+j} p\left(e^{-1 / m}\right) \int_{0}^{1} \frac{x^{j-1} d x}{p\left(x e^{-1 / m}\right)} \\
& =\frac{d^{+} e}{m+j} p\left(e^{-1 / m}\right) e^{j / m} \int_{0}^{e^{-1 / m}} \frac{x^{j-1} d x}{p(x)} \ll \frac{1}{j^{2}},
\end{aligned}
$$

for $j \geqslant m$. Here we have used the fact that $v_{m, j}$ are non-negative (23) and applied Lemma 5 .

Proof of theorem 1] The first step of the proof is to express the coefficients $a_{k}$ in terms of a linear combination of quantities $S(g ; j)$. By the definition of quantity $S(g ; j)$ we see that its generating function can be expressed as

$$
\sum_{j=1}^{\infty} S(g ; j) z^{j}=z g^{\prime}(z) p(z) .
$$

Dividing both sides of the above identity by $p(z)$ we obtain an expression of derivative $g^{\prime}(z)$ as a product of two functions

$$
q(z) \sum_{j=1}^{\infty} S(g ; j) z^{j}=z g^{\prime}(z),
$$

where

$$
q(z)=\frac{1}{p(z)}=\sum_{j=0}^{\infty} q_{j} z^{j},
$$

which is equivalent to the identity expressing the Taylor coefficient $m a_{m}$ of the derivative $g^{\prime}(z)$ as a convolution

$$
k a_{k}=\sum_{j=1}^{k} S(g ; j) q_{k-j} .
$$

We can now use this identity to replace coefficients $a_{k}$ by a sum $(1 / k) \sum_{j=1}^{k} S(g ; j) q_{k-j}$ in the expression appearing on the left hand side of the inequality (5) of our theo- 
rem

$$
\begin{aligned}
& \sum_{k=0}^{n} a_{k} p_{n-k}-p_{n} g\left(e^{-1 / n}\right) \\
& =\sum_{k=1}^{n} p_{n-k} \frac{1}{k} \sum_{j=1}^{k} S(g ; j) q_{k-j}-p_{n} \sum_{k=1}^{\infty} \frac{e^{-k / n}}{k} \sum_{j=1}^{k} S(g ; j) q_{k-j} \\
& =\sum_{j=1}^{n} S(g ; j) \sum_{k=j}^{n} \frac{p_{n-k} q_{k-j}}{k}-p_{n} \sum_{j=1}^{\infty} S(g ; j) \sum_{k=j}^{\infty} \frac{e^{-k / n}}{k} q_{k-j} \\
& =\frac{S(g ; n)}{n}+\sum_{j=1}^{n-1} S(g ; j) \sum_{s=0}^{n-j} \frac{p_{n-j-s} q_{s}}{s+j}-p_{n} \sum_{j=1}^{\infty} S(g ; j) \sum_{k=j}^{\infty} \frac{e^{-k / n}}{k} q_{k-j} .
\end{aligned}
$$

recalling that

$$
v_{m, j}=\sum_{s=0}^{m} \frac{p_{m-s} q_{s}}{s+j}=\int_{0}^{1} g_{m, x} x^{j-1} d x
$$

obtain an identity

$$
\begin{aligned}
\sum_{k=0}^{n} a_{k} p_{n-k}- & p_{n} g\left(e^{-1 / n}\right)-\frac{S(g ; n)}{n} \\
=\sum_{j=1}^{n-1} S(g ; j)\left(v_{n-j, j}-p_{n}\right. & \left.\int_{0}^{e^{-1 / n}} \frac{x^{j-1}}{p(x)} d x\right) \\
& \quad+p_{n} \sum_{j=n}^{\infty} S(g ; j) \int_{0}^{e^{-1 / n}} \frac{x^{j-1}}{p(x)} d x .
\end{aligned}
$$

For brevity, let us denote by $R_{n}$ the quantity on the left hand side of the above identity

$$
R_{n}=\sum_{k=0}^{n} a_{k} p_{n-k}-p_{n} g\left(e^{-1 / n}\right)-\frac{S(g ; n)}{n} .
$$

Then we have

$$
\begin{aligned}
\left|R_{n}\right| \leqslant & \sum_{1 \leqslant j \leqslant n / 2}|S(g ; j)|\left|\int_{0}^{1} x^{j-1} g_{n-j, x} d x-p_{n-j} \int_{0}^{e^{-1 / n}} \frac{x^{j-1}}{p(x)} d x\right| \\
& +\sum_{1 \leqslant j \leqslant n / 2}|S(g ; j)|\left|p_{n}-p_{n-j}\right| \int_{0}^{e^{-1 / n}} \frac{x^{j-1}}{p(x)} d x \\
& +\sum_{n / 2 \leqslant j \leqslant n-1}|S(g ; j)| v_{n-j, j}+p_{n} \sum_{j \geqslant n / 2}|S(g ; j)| \int_{0}^{e^{-1 / n}} \frac{x^{j-1}}{p(x)} d x .
\end{aligned}
$$


Since by (21) we have $g_{m, x} \leqslant e d^{+} \frac{G_{j}\left(e^{-1 / m}\right)}{m}=\frac{e d^{+}}{m} \frac{p\left(e^{-1 / m}\right)}{p\left(e^{-1 / m} x\right)}$, therefore

$$
\begin{aligned}
\int_{e^{-1 / n}}^{1} x^{j-1} g_{n-j, x} d x & \leqslant \frac{e d^{+} p\left(e^{-1 /(n-j)}\right)}{n-j} \int_{e^{-1 / n}}^{1} \frac{d x}{p\left(x e^{-1 /(n-j)}\right)} \\
& \ll \frac{p\left(e^{-1 / n}\right)}{n^{2}} \frac{1}{p\left(e^{-1 / n}\right)}=\frac{1}{n^{2}},
\end{aligned}
$$

when $j \leqslant n / 2$.

This estimate allows us to evaluate the part of the integral of $x^{j-1} g_{n-j, x}$ over interval $e^{-1 / n} \leqslant x \leqslant 1$ in the first sum on the right hand side of inequality (24), making the application of Lemma 6 possible to estimate the difference of integrals in the first sum. The second sum in the inequality (24) can be evaluated using upper bound for difference $\left|p_{n}-p_{n-j}\right|$ of Lemma 2 and the estimate of integral $\int_{0}^{e^{-1 / n}} \frac{x^{j-1}}{p(x)} d x \ll \frac{1}{j p\left(e^{-1 / j}\right)}$ provided by Lemma 5. Finally, applying the estimate $v_{n-j, j} \ll 1 / j^{2}$ of Lemma 7 that is valid in the region $j \geqslant n / 2$ to evaluate the third sum and the upper bound $\int_{0}^{e^{-1 / n}} \frac{x^{j-1}}{p(x)} d x \ll \frac{e^{-j / n}}{j p\left(e^{-1 / n}\right)}$ of Lemma 5 to evaluate the fourth sum in (24) our inequality for $\left|R_{n}\right|$ becomes

$$
\begin{aligned}
\left|R_{n}\right| \ll & \sum_{1 \leqslant j \leqslant n / 2}|S(g ; j)| \frac{p\left(e^{-1 /(n-j)}\right)}{n-j}\left(\frac{j^{\theta-1}}{(n-j)^{\theta}}\right) \frac{1}{p\left(e^{-1 / j}\right)} \\
& +\sum_{1 \leqslant j \leqslant n / 2}|S(g ; j)| p_{n}\left(\frac{j}{n}\right)^{\theta} \frac{1}{j p\left(e^{-1 / j}\right)}+\frac{1}{n^{2}} \sum_{n / 2 \leqslant j \leqslant n-1}|S(g ; j)| \\
& +p_{n} \sum_{j>n / 2}|S(g ; j)| \frac{e^{-j / n}}{j p\left(e^{-1 / n}\right)} \\
& \ll p_{n}\left(\frac{1}{n^{\theta}} \sum_{j=1}^{n} \frac{|S(g ; j)|}{p\left(e^{-1 / j}\right)} j^{\theta-1}+\frac{1}{p\left(e^{-1 / n}\right)} \sum_{j>n} \frac{|S(g ; j)|}{j} e^{-j / n}\right) .
\end{aligned}
$$

The theorem is proved.

Proof of theorem 2, 1) Sufficiency. The second condition (7) of the theorem means that $S(g ; n)=o\left(n p_{n}\right)$. Applying this asymptotic to the right hand side of the inequality of Theorem 1 we get an estimate

$$
\frac{1}{p_{n}} \sum_{k=0}^{n} a_{k} p_{n-k}=g\left(e^{-1 / n}\right)+o(1) .
$$

By the first condition (6) of the theorem $g(x) \rightarrow A$ as $x \uparrow 1$, which means that the right hand of the above estimate converges to $A$ as $n \rightarrow \infty$. This proves that 
conditions (6) and (7) of the theorem imply that

$$
\lim _{n \rightarrow \infty} \frac{1}{p_{n}} \sum_{k=0}^{n} a_{k} p_{n-k}=A .
$$

2) Necessity. Suppose now that limit (25) exists. Let us denote

$$
r_{n}=\sum_{k=0}^{n} a_{k} p_{n-k}
$$

Our assumption (25) means that $r_{n}=A p_{n}\left(1+\varepsilon_{n}\right)$, where $\varepsilon_{n} \rightarrow \infty$ as $n \rightarrow \infty$.

We can express the generating function of $S(g ; n)$ in terms of generating functions of quantities $r_{n}$ and $d_{n}$ as

$$
\begin{aligned}
\sum_{j=1}^{\infty} S(g ; j) z^{j} & =z g^{\prime}(z) p(z)=z(p(z) g(z))^{\prime}-z p^{\prime}(z) g(z) \\
& =z(p(z) g(z))^{\prime}-p(z) g(z) \sum_{k=1}^{\infty} d_{k} z^{k} \\
& =\sum_{n=1}^{\infty} n r_{n} z^{n}-\sum_{n=1}^{\infty} r_{n} z^{n} \sum_{k=1}^{\infty} d_{k} z^{k}
\end{aligned}
$$

Equating the coefficients in the Taylor expansion of the series on the both sides of the above equation we obtain an equation expressing $S(g ; n)$ in terms of $r_{j}$ as

$$
S(g ; n)=n r_{n}-\sum_{k=1}^{n} d_{k} r_{n-k}
$$

Inserting here $r_{n}=A p_{n}\left(1+\varepsilon_{n}\right)$ we obtain

$$
\begin{aligned}
S(g ; n) & =A n p_{n}-A \sum_{k=1}^{n} d_{k} p_{n-k}+A n p_{n} \varepsilon_{n}-A \sum_{k=1}^{n} d_{k} p_{n-k} \varepsilon_{n-k} \\
& =A n p_{n} \varepsilon_{n}-A \sum_{k=1}^{n} d_{k} p_{n-k} \varepsilon_{n-k}=o\left(n p_{n}\right),
\end{aligned}
$$

since $n p_{n}=\sum_{k=1}^{n} d_{k} p_{n-k}$ and $n p_{n} \gg p\left(e^{-1 / n}\right) \rightarrow \infty$ as $n \rightarrow \infty$.

The necessity of condition 2) is proved.

The necessity of condition 1) is well known, see e. g. [6]. It is obtained by noticing that

$$
g(x)=\frac{g(x) p(x)}{p(x)}=\frac{r_{0}+r_{2} x+\cdots+r_{n} x^{n}+\cdots}{p_{0}+p_{2} x+\cdots+p_{n} x^{n}+\cdots} .
$$


Using estimate $r_{n}=A p_{n}(1+o(1))$ to evaluate the right hand side of the above equation and taking into account the fact that $p(x) \rightarrow \infty$ as $x \nearrow 1$ we conclude that the left hand side of the above equation has a limit

$$
g(x) \rightarrow A
$$

as $x \nearrow 1$.

The theorem is proved.

\subsection{Random permutations}

Recall that if we denote by $\alpha_{k}(\sigma)$ the number of cycles in permutation $\sigma$ whose length is equal to $k$ then the value of multiplicative function $f(\sigma)$ can be expressed as a product

$$
f(\sigma)=\hat{f}(1)^{\alpha_{1}(\sigma)} \hat{f}(2)^{\alpha_{2}(\sigma)} \ldots \hat{f}(n)^{\alpha_{n}(\sigma)},
$$

where we assume that $0^{0}=1$ in the above relationship.

It can be proved by elementary combinatorial arguments (see e.g. [2], page 233, Theorem B) that the quantity of permutations $\sigma \in S_{n}$ such that $\alpha_{j}(\sigma)=k_{j}$ for $1 \leqslant j \leqslant n$ is equal to

$$
n ! \prod_{j=1}^{n} \frac{1}{k_{j} ! j^{k_{j}}}
$$

when $k_{1}+2 k_{2}+\cdots+n k_{n}=n$. This fact allows us to express the sum of values $f(\sigma)$ of multiplicative function $f$ over all permutations $\sigma \in S_{n}$ as

$$
\sum_{\sigma \in S_{n}} f(\sigma)=n ! \sum_{k_{1}+2 k_{2}+\cdots+n k_{n}=n} \prod_{j=1}^{n}\left(\frac{\hat{f}(j)}{j}\right)^{k_{j}} \frac{1}{k_{j} !} .
$$

The above expression leads to the identity of the corresponding generating functions

$$
1+\sum_{m=1}^{\infty}\left(\frac{1}{m !} \sum_{\sigma \in S_{m}} f(\sigma)\right) z^{m}=\prod_{j \geqslant 1} \sum_{k=0}^{\infty}\left(\frac{\hat{f}(j)}{j}\right)^{k} \frac{z^{j k}}{k !}=\exp \left\{\sum_{j=1}^{\infty} \frac{\hat{f}(j)}{j} z^{j}\right\} .
$$

This in its turn leads to the expression for the mean value of a multiplicative function

$$
M_{n}^{d}(f)=\frac{\sum_{\sigma \in S_{n}} f(\sigma) d(\sigma)}{\sum_{\sigma \in S_{n}} d(\sigma)}=\frac{\left[z^{n}\right] \exp \left\{\sum_{j=1}^{\infty} \frac{\hat{d}(j) \hat{f}(j)}{j} z^{j}\right\}}{\left[z^{n}\right] \exp \left\{\sum_{j=1}^{\infty} \frac{\hat{d}(j)}{j} z^{j}\right\}}
$$


as a ratio of coefficients of appropriate generating functions. Let us denote the generating functions appearing in the above expression of the mean value $M_{n}^{d}(f)$ as

$$
M(z)=\sum_{j=0}^{\infty} M_{j} z^{j}=\exp \left\{\sum_{j=1}^{\infty} \frac{\hat{d}(j) \hat{f}(j)}{j} z^{j}\right\}
$$

and

$$
p(z)=\sum_{j=0}^{\infty} p_{j} z^{j}=\exp \left\{\sum_{j=1}^{\infty} \frac{\hat{d}(j)}{j} z^{j}\right\} .
$$

Since later we will apply Theorem 1 with $\hat{d}(j)=d_{j}$, so from now on we will identify the quantity $\hat{d}(j)$ with $d_{j}$. We also will use the same notation $p(z)$ for the function $\exp \left\{\sum_{j=1}^{\infty} \frac{\hat{d}(j)}{j} z^{j}\right\}$ as for the for function $\exp \left\{\sum_{j=1}^{\infty} \frac{d_{j}}{j} z^{j}\right\}$.

We can represent the generating function $M(z)$ as a product of two functions

$$
\begin{aligned}
M(z) & =\exp \left\{\sum_{j=1}^{\infty} \frac{\hat{d}(j)}{j} z^{j}\right\} \exp \left\{\sum_{j=1}^{\infty} \hat{d}(j) \frac{\hat{f}(j)-1}{j} z^{j}\right\} \\
& =p(z) m(z),
\end{aligned}
$$

where

$$
m(z)=\sum_{j=0}^{\infty} m_{j} z^{j}=\exp \left\{\sum_{j=1}^{\infty} \hat{d}(j) \frac{\hat{f}(j)-1}{j} z^{j}\right\} .
$$

With these notations we can express the mean value of a multiplicative function as

$$
M_{n}^{d}(f)=\frac{\left[z^{n}\right] p(z) m(z)}{\left[z^{n}\right] p(z)}=\frac{M_{n}}{p_{n}}=\frac{1}{p_{n}} \sum_{j=0}^{n} m_{j} p_{n-j} .
$$

Before going into the details of proof of Theorem 3 let us at first illustrate the ideas of application of Tauberian theory for Voronoi summation for the analysis of mean values $M_{n}^{d}(f)$ on a simple example.

Proposition 1. Suppose we are given a fixed sequence $\hat{f}(1), \hat{f}(2), \ldots, \hat{f}(n), \ldots$ of complex numbers, such that the modulus of the members of this sequence does not exceed one $|\hat{f}(j)| \leqslant 1$ and

$$
\lim _{k \rightarrow \infty} \hat{f}(k)=1
$$

Then the sequence of mean values of the corresponding multiplicative function $f(\sigma)$ has the following asymptotic

$$
M_{n}^{d}(f)=\frac{\sum_{\sigma \in S_{n}} f(\sigma) d(\sigma)}{\sum_{\sigma \in S_{n}} d(\sigma)}=\exp \left\{\sum_{j=1}^{\infty} \hat{d}(j) \frac{\hat{f}(j)-1}{j} e^{-j / n}\right\}+o(1),
$$


as $n \rightarrow \infty$.

Proof. We have already shown that the generating function $M(z)$ is a product of two functions $M(z)=p(z) m(z)$. By our results on Voronoi means, if we can prove that Tauberian condition

$$
\left[z^{n-1}\right] p(z) m^{\prime}(z)=\sum_{k=0}^{n} k m_{k} p_{n-k}=o\left(n p_{n}\right)
$$

holds, then

$$
\left[z^{n}\right] p(z) m(z)=p_{n}\left(m\left(e^{-1 / n}\right)+o(1)\right) \quad \text { as } \quad n \rightarrow \infty .
$$

which will imply an estimate for the mean value

$$
M_{n}^{d}(f)=\frac{\left[z^{n}\right] p(z) m(z)}{\left[z^{n}\right] p(z)}=m\left(e^{-1 / n}\right)+o(1) .
$$

To check the Tauberian condition (28) we note that

$$
p(z) m^{\prime}(z)=p(z) m(z) \sum_{j=1}^{\infty} \hat{d}(j) \frac{\hat{f}(j)-1}{j} z^{j-1}=M(z) \sum_{j=1}^{\infty} \hat{d}(j) \frac{\hat{f}(j)-1}{j} z^{j-1}
$$

which means that

$$
S(m ; n)=\left[z^{n-1}\right] p(z) m^{\prime}(z)=\sum_{j=1}^{n} \hat{d}(j)(\hat{f}(j)-1) M_{n-j} .
$$

Since $|\hat{f}(j)| \leqslant 1$ we have

$$
\begin{aligned}
\left|M_{k}\right| & =\left|\left[z^{k}\right] \exp \left\{\sum_{j=1}^{\infty} \frac{d_{j} \hat{f}(j)}{j} z^{j}\right\}\right|=\left|\sum_{\ell_{1}+2 \ell_{2}+\cdots+k \ell_{k}=k} \prod_{j=1}^{k}\left(\frac{d_{j} \hat{f}(j)}{j}\right)^{\ell_{j}} \frac{1}{\ell_{j} !}\right| \\
& \leqslant \sum_{\ell_{1}+2 \ell_{2}+\cdots+k \ell_{k}=k} \prod_{j=1}^{k}\left(\frac{d_{j}}{j}\right)^{\ell_{j}} \frac{1}{\ell_{j} !}=\left[z^{k}\right] \exp \left\{\sum_{j=1}^{\infty} \frac{d_{j}}{j} z^{j}\right\}=p_{k} .
\end{aligned}
$$

Applying inequalities $\left|M_{k}\right| \leqslant p_{k}$ and $d_{j}=\hat{d}(j) \leqslant d^{+}$to estimate the right hand side of the identity (29) we conclude that in order to show that $S(m ; n)=o\left(n p_{n}\right)$ it is enough to check that

$$
\sum_{j=1}^{n}|\hat{f}(j)-1| p_{n-j}=o\left(n p_{n}\right)
$$


From the recurrence relations (16) for $p_{n}$ we obtain an inequality

$$
p_{j}=\frac{1}{j} \sum_{k=1}^{j} d_{k} p_{j-k} \leqslant \frac{d^{+}}{j} \sum_{k=0}^{j-1} p_{k} \leqslant \frac{d^{+}}{d^{-} j} \sum_{k=1}^{n} d_{k} p_{n-k}=\frac{d^{+} n}{d^{-} j} p_{n}
$$

for any $1 \leqslant j \leqslant n$. This inequality enables us to show that a finite number of the first summands of the sum of (30) is negligible. That is, for any fixed $T<n$ the inequality

$$
\begin{aligned}
\sum_{j=1}^{n}|\hat{f}(j)-1| p_{n-j} & \leqslant \frac{2 d^{+} n p_{n}}{d^{-}} \sum_{j \leqslant T} \frac{1}{n-j}+\sup _{j \geqslant T}|\hat{f}(j)-1| \sum_{j=1}^{n} p_{n-j} \\
& \leqslant \frac{2 n p_{n} d^{+} T}{d^{-}(n-T)}+\sup _{j \geqslant T}|\hat{f}(j)-1| \frac{n p_{n}}{d^{-}}
\end{aligned}
$$

holds. By the condition of our proposition $\sup _{j \geqslant T}|\hat{f}(j)-1| \rightarrow 0$ as $T \rightarrow \infty$. Therefore if we chose, for example $T:=\sqrt{n}$, then the second term of the sum in the last inequality will be $o\left(n p_{n}\right)$ while the first term will be of order $O\left(\sqrt{n} p_{n}\right)=$ $o\left(n p_{n}\right)$. This proves that sum of both terms is $o\left(n p_{n}\right)$.

A number of conclusions can be drawn from the just proven proposition. First of all it is clear that if multiplicative function $f(\sigma)$ satisfies the conditions of the proposition, then the mean value has a zero limit

$$
\lim _{n \rightarrow \infty} M_{n}^{d}(f)=0
$$

if and only if diverges the series

$$
\sum_{j=1}^{\infty} \frac{1-\Re \hat{f}(j)}{j}=\infty .
$$

The case of convergence of the above series can be split into two cases. The existence of the non-zero $\operatorname{limit}_{n \rightarrow \infty} M_{n}^{d}(f) \neq 0$ is equivalent to convergence of the series

$$
\sum_{j=1}^{\infty} d_{j} \frac{\hat{f}(j)-1}{j}
$$

If the imaginary part of the above series diverges then $M_{n}^{d}(f)=c(f) e^{i L(n)}+o(1)$,

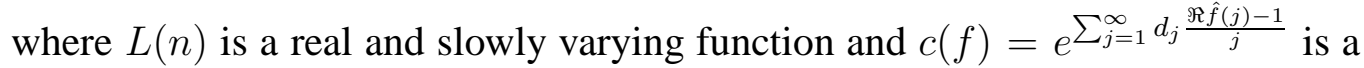
positive constant.

The result of Proposition 1 and its consequences are not new. They follow from more general results of Manstavičius [10] that were obtained by using a different approach. 
Proof of Theorem 3 Since $\hat{f}(k)$ for $k>n$ do not influence the $n$-th Taylor coefficient $M_{n}$ of the generating function $M(z)$, we assume that $\hat{f}(k)=1$ for $k>n$. In the proof of the previous Proposition we have already obtained inequality

$$
|S(m ; j)|=\left|\left[z^{j-1}\right] p(z) m^{\prime}(z)\right| \leqslant d^{+} \sum_{k=1}^{j}|\hat{f}(k)-1| p_{j-k} .
$$

Using this estimate to bound the right hand side of the inequality of Theorem 1 with $g(z)=m(z)$ we obtain inequality

$$
\begin{array}{r}
\left|\frac{M_{n}}{p_{n}}-m\left(e^{-1 / n}\right)\right|=\left|\frac{M_{n}}{p_{n}}-\exp \left\{\sum_{k=1}^{n} \frac{d_{k}(\hat{f}(k)-1)}{k} e^{-k / n}\right\}\right| \\
\leqslant c\left(\frac{1}{n p_{n}} \sum_{k=1}^{n}|\hat{f}(k)-1| p_{n-k}+\frac{1}{n^{\theta}} \sum_{j=1}^{n} \frac{j^{\theta-1}}{p\left(e^{-1 / j}\right)} \sum_{k=1}^{j}|\hat{f}(k)-1| p_{j-k}\right. \\
\left.+\frac{1}{p\left(e^{-1 / n}\right)} \sum_{j>n} \frac{e^{-j / n}}{j} \sum_{k=1}^{j}|\hat{f}(k)-1| p_{j-k}\right),
\end{array}
$$

where $\theta=\min \left\{1, d^{-}\right\}$and $c=c\left(d^{+}, d^{-}\right)$. The inequality in the formulation of the theorem will follow after we find simpler estimates for the second and the third term in the sum on the right hand side of the above inequality. Let us start with the second term. Changing the order of summation we get

$$
\begin{aligned}
& \frac{1}{n^{\theta}} \sum_{j=1}^{n} \frac{j^{\theta-1}}{p\left(e^{-1 / j}\right)} \sum_{k=1}^{j}|\hat{f}(k)-1| p_{j-k}=\frac{1}{n^{\theta}} \sum_{k=1}^{n}|\hat{f}(k)-1| \sum_{n \geqslant j \geqslant k} \frac{j^{\theta-1}}{p\left(e^{-1 / j}\right)} p_{j-k} \\
& \ll \frac{1}{n^{\theta}} \sum_{k=1}^{n}|\hat{f}(k)-1| \sum_{n \geqslant j \geqslant 2 k} \frac{j^{\theta-1}}{p\left(e^{-1 / j}\right)} \frac{p\left(e^{-1 /(j-k)}\right)}{j-k} \\
& \quad+\frac{1}{n^{\theta}} \sum_{k=1}^{n}|\hat{f}(k)-1| k^{\theta-1} \sum_{2 k \geqslant j \geqslant k} \frac{p_{j-k}}{p\left(e^{-1 / j}\right)} .
\end{aligned}
$$

As a Taylor series with positive coefficients function $p(x)$ is increasing for increasing values of $x$. Therefore for $j>k$ we have $p\left(e^{-1 /(j-k)}\right) \leqslant p\left(e^{-1 / j}\right)$ and 
$p\left(e^{-1 / j}\right) \geqslant p\left(e^{-1 / k}\right)$. Using these inequalities to evaluate the last estimate we get

$$
\begin{aligned}
& \frac{1}{n^{\theta}} \sum_{j=1}^{n} \frac{j^{\theta-1}}{p\left(e^{-1 / j}\right)} \sum_{k=1}^{j}|\hat{f}(k)-1| p_{j-k} \\
& \ll \frac{1}{n^{\theta}} \sum_{k=1}^{n}|\hat{f}(k)-1| \sum_{n \geqslant j \geqslant 2 k} j^{\theta-2}+\frac{1}{n^{\theta}} \sum_{k=1}^{n}|\hat{f}(k)-1| k^{\theta-1} \frac{1}{p\left(e^{-1 / k}\right)} \sum_{s=0}^{k} p_{s} \\
& \ll \frac{1}{n^{\theta}} \sum_{k=1}^{n}|\hat{f}(k)-1|\left(k^{\theta-1}+\int_{k}^{n} x^{\theta-2} d x\right)
\end{aligned}
$$

The third term can be handled in a similar way

$$
\begin{gathered}
\frac{1}{p\left(e^{-1 / n}\right)} \sum_{j>n} \frac{e^{-j / n}}{j} \sum_{k=1}^{n}|\hat{f}(k)-1| p_{j-k}=\frac{1}{p\left(e^{-1 / n}\right)} \sum_{k=1}^{n}|\hat{f}(k)-1| \sum_{j>n} \frac{e^{-j / n}}{j} p_{j-k} \\
\leqslant \frac{1}{n} \sum_{k=1}^{n}|\hat{f}(k)-1| \frac{e^{-k / n}}{p\left(e^{-1 / n}\right)} \sum_{j>n} e^{-(j-k) / n} p_{j-k} \leqslant \frac{1}{n} \sum_{k=1}^{n}|\hat{f}(k)-1| .
\end{gathered}
$$

The statement of the theorem will follow if we use inequality

$$
\left|\sum_{k=1}^{n} \frac{d_{k}(\hat{f}(k)-1)}{k} e^{-k / n}-\sum_{k=1}^{n} \frac{d_{k}(\hat{f}(k)-1)}{k}\right| \leqslant \frac{1}{n} \sum_{k=1}^{n} d_{k}|\hat{f}(k)-1|
$$

to estimate the quantity under exponent in our inequality (31).

The theorem is proved.

Let us define

$$
L_{n}(z)=\sum_{j=1}^{n} d_{j} \frac{\hat{f}(j)-1}{j} z^{j} \quad \text { and } \quad \rho_{n}(p)=\left(\sum_{j=1}^{n} \frac{|\hat{f}(j)-1|^{p}}{j}\right)^{1 / p}
$$

moreover we assume that

$$
\rho_{n}(\infty)=\lim _{p \rightarrow \infty} \rho_{n}(p)=\max _{1 \leqslant k \leqslant n}|\hat{f}(k)-1| \quad \text { and } \quad \rho(p)=\left(\sum_{j=1}^{\infty} \frac{|\hat{f}(j)-1|^{p}}{j}\right)^{1 / p},
$$

likewise we will write $L(z)=\sum_{j=1}^{\infty} d_{j} \frac{\hat{f}(j)-1}{j} z^{j}$. For brevity, we will often write simply $\rho$ instead of $\rho(p)$ if the value of $p$ is known from the context. 
Lemma 8. For $n, m \geqslant 1$ and $1 / p+1 / q=1$ with $\infty \geqslant p>1$, we have

$$
\left|L\left(e^{-1 / n}\right)-L\left(e^{-1 / m}\right)\right| \leqslant d^{+} \rho(p)\left(1+\left|\log \frac{n}{m}\right|\right)
$$

and

$$
\left|L\left(e^{-1 / n}\right)\right| \leqslant d^{+} \rho(p)(1+\log n) .
$$

Proof. Without loss of generality we can assume that $n \geqslant m \geqslant 1$. Suppose first that $p<\infty$. Applying Cauchy's inequality with parameters $p, q$ we have

$$
\begin{aligned}
\left|L\left(e^{-\frac{1}{n}}\right)-L\left(e^{-\frac{1}{m}}\right)\right| & \leqslant d^{+} \sum_{j=1}^{\infty} \frac{|\hat{f}(j)-1|}{j}\left|e^{-j / n}-e^{-j / m}\right| \\
& \leqslant d^{+} \rho(p)\left(\sum_{j=1}^{\infty} \frac{\left|e^{-j / n}-e^{-j / m}\right|^{q}}{j}\right)^{1 / q} \\
& \leqslant d^{+} \rho(p)\left(\sum_{j=1}^{\infty} \frac{e^{-j / n}-e^{-j / m}}{j}\right)^{1 / q} \\
& =d^{+} \rho(p)\left(\log \frac{1-e^{-1 / m}}{1-e^{-1 / n}}\right)^{1 / q} \\
& \leqslant d^{+} \rho(p)\left(\frac{1}{n}+\log \frac{n}{m}\right)^{1 / q}
\end{aligned}
$$

in the last step we used inequality $e^{-x} x<1-e^{-x}<x$, which is true for all $x>0$, to estimate the ratio of quantities $\frac{1-e^{-1 / m}}{1-e^{-1 / n}}$ under the sign of logarithm. Finally estimating the fraction $1 / \mathrm{m}$ in the last expression by means of a crude upper bound $\frac{1}{m} \leqslant 1$ and taking into account that $1 / q \leqslant 1$ we obtain the first inequality in the statement of the lemma for finite values of $p>1$. Allowing $p \rightarrow \infty$ we see that this inequality is true for $p=\infty$ also. Similar considerations lead to the second inequality.

Lemma 9. For all $n \geqslant 1$ and $\varepsilon \geqslant 0, q \geqslant 1$ fixed such that $q\left(d^{-}-1\right)-\varepsilon>-1$, then

$$
\sum_{j=1}^{n} j^{-\varepsilon} p_{j}^{q} \ll n^{1-\varepsilon} p_{n}^{q} \quad \text { and } \quad \sum_{j=1}^{n} \frac{1}{j}\left|\frac{p_{n-j}}{p_{n}}-1\right|^{q} \ll 1 .
$$

Proof. Applying the upper (17) and lower (18) bounds for $p_{j}$ and using the esti- 
mate for ratio $\frac{p\left(e^{-1 / j}\right)}{p\left(e^{-1 / n}\right)}$ provided by Lemma 1 we obtain

$$
\begin{aligned}
\sum_{j=1}^{n} j^{-\varepsilon} p_{j}^{q} & \leqslant \sum_{j=1}^{n} \frac{1}{j^{\varepsilon}}\left(e d^{+} \frac{p\left(e^{-1 / j}\right)}{j}\right)^{q}=\left(e d^{+}\right)^{q}\left(\frac{p\left(e^{-1 / n}\right)}{n}\right)^{q} \sum_{j=1}^{n} \frac{1}{j^{\varepsilon}}\left(\frac{n p\left(e^{-1 / j}\right)}{j p\left(e^{-1 / n}\right)}\right)^{q} \\
& \ll\left(\frac{p\left(e^{-1 / n}\right)}{n}\right)^{q} \sum_{j \leqslant n} \frac{1}{j^{\varepsilon}}\left(\frac{j}{n}\right)^{q\left(d^{-}-1\right)} \ll n^{1-\varepsilon} p_{n}^{q} .
\end{aligned}
$$

We will estimate the second sum by splitting it into two parts

$$
\sum_{j=1}^{n} \frac{1}{j}\left|\frac{p_{n-j}}{p_{n}}-1\right|^{q} \ll \sum_{1 \leqslant j \leqslant n / 4} \frac{1}{j}\left|\frac{p_{n-j}-p_{n}}{p_{n}}\right|^{q}+\frac{1}{n} \sum_{n / 4<j \leqslant n}\left(\left|\frac{p_{n-j}}{p_{n}}\right|^{q}+1\right) .
$$

The second sum in the last inequality is $O(1)$ by the just proven estimate for the sum of $p_{j}^{q}$. While the first sum can be estimated applying the upper bound for difference $p_{n-j}-p_{n}$ provided by Lemma2, which yields

$$
\sum_{j \leqslant n / 4} \frac{1}{j}\left|\frac{p_{n-j}-p_{n}}{p_{n}}\right|^{q} \ll \sum_{1 \leqslant j \leqslant n / 4} \frac{1}{j}\left|\frac{j}{n}\right|^{q \theta} \ll 1 .
$$

The lemma is proved.

Proposition 2. Consider a sequence of complex numbers $\hat{f}(1), \hat{f}(2), \ldots, \hat{f}(n), \ldots$, such that $|\hat{f}(j)| \leqslant 1$ then for any fixed $p>1$ there is a positive constant $c_{3}=$ $c_{3}\left(p, d^{-}, d^{+}\right)$such that

$$
\left|\frac{M_{n}}{p_{n}}-m\left(e^{-1 / n}\right)\right| \leqslant c_{3}\left(\sum_{j=1}^{\infty} \frac{|\hat{f}(j)-1|^{p}}{j}\right)^{1 / p} \quad\left(=c_{3} \rho(p)\right)
$$

for all $n \geqslant 1$.

Proof. If the series on the right hand side of the inequality of our proposition diverges, then the proposition becomes trivial. Therefore let us assume that this series is convergent. Applying the estimate of sums of $p_{j}^{q}$ of Lemma 9 we obtain inequality

$$
\begin{aligned}
|S(m ; n)| & \leqslant \sum_{j=1}^{n} \hat{d}(j)|\hat{f}(j)-1| p_{n-j} \leqslant d^{+}\left(\sum_{j=1}^{n} p_{n-j}^{q}\right)^{1 / q}\left(\sum_{j=1}^{n}|\hat{f}(j)-1|^{p}\right)^{1 / p} \\
& \ll n p_{n}\left(\sum_{j=1}^{n} \frac{|\hat{f}(j)-1|^{p}}{n}\right)^{1 / p} \leqslant n p_{n}\left(\sum_{j=1}^{\infty} \frac{|\hat{f}(j)-1|^{p}}{j}\right)^{1 / p}
\end{aligned}
$$

Applying this inequality to estimate the right hand side of inequality Theorem 1 for difference $\frac{M_{n}}{p_{n}}-m\left(e^{-1 / n}\right)$, we complete the proof of the Proposition. 
Unfortunately the estimate of the just proven proposition is not strong enough for our purpose as we will need an estimate like $O\left(\rho(p) m\left(e^{-1 / n}\right)\right)$ in order to analyze characteristic functions of additive functions.

Proposition 3. For any fixed $\infty \geqslant p>\max \left\{1,1 / d^{-}\right\}$, there exists such a positive $\delta=\delta\left(d^{-}, d^{+}, p\right)$ that if $\rho=\rho(p) \leqslant \delta$, then

$$
\frac{M_{n}}{p_{n}}=m\left(e^{-1 / n}\right)(1+O(\rho)) .
$$

Proof. At first let us prove that $M_{n}=O\left(p_{n}\left|m\left(e^{-1 / n}\right)\right|\right)$ for all $n \geqslant 0$ (here and in what follows we assume that $e^{-1 / 0}=0$ ). Let us assume that only finite number of $\hat{f}(j)$ are not equal to 1 . Then the supremum $D$ of ratios

$$
D:=\sup _{n \geqslant 0}\left|\frac{M_{n}}{p_{n} m\left(e^{-1 / n}\right)}\right|
$$

will be finite. We will prove that $D \leqslant 2$ if $\rho(p) \leqslant \delta$, with some absolute, sufficiently small constant $\delta>0$. Let us use inequality $\left|M_{k}\right| \leqslant D p_{k}\left|m\left(e^{-1 / k}\right)\right|$ to estimate the right hand side of the identity (29) for $S(m ; n)$ as

$$
\begin{aligned}
|S(m ; n)| & \leqslant D d^{+} \sum_{j=1}^{n}|\hat{f}(j)-1| p_{n-j}\left|m\left(e^{-1 /(n-j)}\right)\right| \\
& \leqslant D d^{+} n\left(\frac{1}{n} \sum_{j=1}^{n}|\hat{f}(j)-1|^{p}\right)^{1 / p}\left(\frac{1}{n} \sum_{j=0}^{n-1} p_{j}^{q}\left|m\left(e^{-1 / j}\right)\right|^{q}\right)^{1 / q} \\
& \leqslant D d^{+} n \rho\left|m\left(e^{-1 / n}\right)\right|\left(\frac{1}{n} \sum_{j=0}^{n-1} p_{j}^{q}\left|\frac{m\left(e^{-1 / j}\right)}{m\left(e^{-1 / n}\right)}\right|^{q}\right)^{1 / q},
\end{aligned}
$$

for $n \geqslant 1$. Since $m\left(e^{-1 / k}\right)=e^{L\left(e^{-1 / k}\right)}$ we can apply Lemma 8 to estimate the ratio

$$
\begin{aligned}
\left|\frac{m\left(e^{-1 / j}\right)}{m\left(e^{-1 / n}\right)}\right| & \leqslant \exp \left\{\left|L\left(e^{-1 / n}\right)-L\left(e^{-1 / j}\right)\right|\right\} \\
& \leqslant \exp \left\{d^{+} \rho(p)\left(1+\log \frac{n}{j}\right)\right\}=e^{d^{+} \rho(p)}\left(\frac{n}{j \vee 1}\right)^{d^{+} \rho(p)}
\end{aligned}
$$

for $n \geqslant j \geqslant 0$, where we use the notation $a \vee b=\max \{a, b\}$. From now on let us assume that $\delta$ is small enough that $q\left(d^{-}-1\right)-q d^{+} \delta>-1$, which is necessary to ensure the validity of the upper bound of Lemma 9 for the partial 
sum of $p_{j}^{q} j^{-q d^{+} \rho(p)}$. This allows us to further evaluate $S(m ; n)$ as

$$
\begin{aligned}
|S(m ; n)| & \leqslant D d^{+} e^{\rho d^{+}} n \rho\left|m\left(e^{-1 / n}\right)\right|\left(\frac{1}{n} \sum_{j=0}^{n-1} p_{j}^{q}\left(\frac{n}{j \vee 1}\right)^{q d^{+} \rho(p)}\right)^{1 / q} \\
& \leqslant C_{1} D \rho n p_{n}\left|m\left(e^{-1 / n}\right)\right|
\end{aligned}
$$

where $C_{1}=C_{1}\left(d^{+}, d^{-}, p\right)$ is a positive constant. Plugging this estimate into the right hand side of the inequality of Theorem 1 we obtain

$$
\begin{aligned}
\left|\frac{M_{n}}{p_{n}}-m\left(e^{-1 / n}\right)\right| \ll & \frac{D \rho}{n^{\theta}} \sum_{j=1}^{n}\left|m\left(e^{-1 / j}\right)\right| j^{\theta-1}+\frac{D \rho}{p\left(e^{-1 / n}\right)} \sum_{j>n}\left|m\left(e^{-1 / j}\right)\right| p_{j} e^{-j / n} \\
& +\rho D\left|m\left(e^{-1 / n}\right)\right|,
\end{aligned}
$$

and once again utilizing the upper bound (32) for ratio $\left|\frac{m\left(e^{-1 / j}\right)}{m\left(e^{-1 / n}\right)}\right|$ and noticing that $\left|m\left(e^{-1 / j}\right)\right|$ is monotonously decreasing as $j$ increases we finally get

$$
\left|\frac{M_{n}}{p_{n}}-m\left(e^{-1 / n}\right)\right| \leqslant C_{2} D \rho(p)\left|m\left(e^{-1 / n}\right)\right|,
$$

if $\delta d^{+}<\theta$, where again $C_{2}$ is a positive constant that depends on $d^{+}, d^{-}$and $p$ only. Dividing both sides of this inequality by $\left|m\left(e^{-1 / n}\right)\right|$, taking maximum for all $n \geqslant 0$ and recalling the definition of $D$ we conclude that this quantity satisfies inequality

$$
D \leqslant 1+C_{2} D \rho(p) .
$$

Thus if we require $\delta$ to be fixed and small enough to ensure that $\rho(p) \leqslant \delta \leqslant$ $1 /\left(2 C_{2}\right)$, then $D$ would be bounded $D \leqslant 2$. Thus if $\delta$ is fixed such that $\delta<$ $\min \left\{\frac{q\left(d^{-}-1\right)+1}{q d^{+}}, \frac{\theta}{d^{+}}, \frac{1}{2 C_{2}}\right\}$ then the estimate of the proposition will follow from the inequality (33) and the fact that $D$ is bounded for such $\delta$. Note that at the beginning of the proof we assumed that only a finite number of $\hat{f}(j)$ are not equal to 1 . However this condition was only needed to ensure that quantity $D$ is finite, all the constants in symbols $O(\ldots)$ and $\ll$ do not depend on the number of $\hat{f}(j)$ that are not equal to 1 . Thus if we have an infinite sequence $\hat{f}(j)$ such that $\rho(p) \leqslant \delta$ then we can consider a modified sequence that is obtained by putting $\hat{f}(j)=1$ for $j \geqslant N$ and allow $N \rightarrow \infty$.

The following theorem has been proved for $d_{j} \equiv 1$ by Manstavičius [11], later generalized for $d_{j} \equiv \theta>0$ in [15]. 
Theorem 6. For any fixed $\infty \geqslant p>\max \left\{1,1 / d^{-}\right\}$, there exists such a positive $\delta=\delta\left(d^{-}, d^{+}, p\right)$ that if $\rho \leqslant \delta$, then

$$
\frac{M_{N}}{p_{N}}=\exp \left\{L_{N}(1)\right\}\left(1+\sum_{j=1}^{N} d_{j} \frac{\hat{f}(j)-1}{j}\left(\frac{p_{N-j}}{p_{N}}-1\right)+O\left(\rho^{2}\right)\right) .
$$

Proof. The values of $\hat{f}(j)$ with $j>N$ do not influence the value of $M_{n}$ therefore we will assume that $\hat{f}(j)=1$ for all $j>N$. Let us consider $S(g ; n)$ with $g(z)=$ $u_{N}(z)=m(z)-m\left(e^{-1 / N}\right) \sum_{j=1}^{\infty} d_{j} \frac{\hat{f}(j)-1}{j} z^{j}$ instead of $g(z)=m(z)$ then

$$
\begin{aligned}
S\left(u_{N} ; n\right) & =\left[z^{n-1}\right] p(z) u_{N}^{\prime}(z) \\
& =S(m ; n)-\left[z^{n-1}\right] m\left(e^{-1 / N}\right) p(z) \sum_{j=1}^{\infty} d_{j}(\hat{f}(j)-1) z^{j-1} \\
& =\sum_{j=1}^{n} d_{j}(\hat{f}(j)-1)\left(M_{n-j}-m\left(e^{-1 / N}\right) p_{n-j}\right)
\end{aligned}
$$

Applying here the estimate $M_{n-j}=p_{n-j} m\left(e^{1 /(n-j)}\right)(1+O(\rho))$ of Proposition 3 after some evaluations we get

$$
\begin{aligned}
S\left(u_{N} ; n\right) \ll & \sum_{j=1}^{n}|\hat{f}(j)-1|\left|m\left(e^{-1 /(n-j)}\right)-m\left(e^{-1 / N}\right)\right| p_{n-j} \\
& +\rho \sum_{j=1}^{n}|\hat{f}(j)-1| p_{n-j}\left|m\left(e^{-1 /(n-j)}\right)\right|
\end{aligned}
$$

The second sum of the above estimate has already been already shown to be $O\left(\rho^{2} n p_{n}\left|m\left(e^{-1 / n}\right)\right|\right)$ in the proof of Proposition 3, Therefore

$$
\begin{aligned}
S\left(u_{N} ; n\right) & \ll n \rho\left(\frac{1}{n} \sum_{j=0}^{n-1}\left|m\left(e^{-1 / j}\right)-m\left(e^{-1 / N}\right)\right|^{q} p_{j}^{q}\right)^{1 / q}+\rho^{2} n p_{n}\left|m\left(e^{-1 / n}\right)\right| \\
& \ll n \rho^{2}\left|m\left(e^{-1 / N}\right)\right|\left(\frac{1}{n} \sum_{j=0}^{n-1} \exp \left\{q d^{+} \rho\left|\log \frac{N}{j \vee 1}\right|\right\} p_{j}^{q}\right)^{1 / q}+\rho^{2} n p_{n}\left|m\left(e^{-1 / n}\right)\right|
\end{aligned}
$$

Since $e^{\beta|\log x|} \leqslant x^{\beta}+x^{-\beta}$ if $\beta>0$, we can further estimate

$$
\begin{aligned}
S\left(u_{N} ; n\right) & \ll n \rho^{2}\left(\frac{1}{n} \sum_{j=0}^{n-1} p_{j}^{q}\left(\left(\frac{N}{j \vee 1}\right)^{q d^{+} \rho}+\left(\frac{j}{N}\right)^{q d^{+} \rho}\right)\right)^{1 / q}+\rho^{2} n p_{n}\left|m\left(e^{-1 / n}\right)\right| \\
& \ll n \rho^{2} p_{n}\left|m\left(e^{-1 / N}\right)\right|\left(\left(\frac{N}{n}\right)^{d^{+} \rho}+\left(\frac{n}{N}\right)^{d^{+} \rho}\right)+\rho^{2} n p_{n}\left|m\left(e^{-1 / n}\right)\right| .
\end{aligned}
$$


After plugging this estimate into the inequality (5) for Voronoi mean we end up with an estimate

$$
\frac{1}{p_{N}}\left[z^{N}\right] p(z) u_{N}(z)-u_{N}\left(e^{-1 / N}\right) \ll \rho^{2}\left|m\left(e^{-1 / N}\right)\right|,
$$

which after recalling the definition of $u_{N}$ becomes

$$
\frac{M_{N}}{p_{N}}=m\left(e^{-1 / N}\right)\left(1+\sum_{j=1}^{N} d_{j} \frac{(\hat{f}(j)-1)}{j}\left(\frac{p_{N-j}}{p_{N}}-e^{-j / N}\right)+O\left(\rho^{2}\right)\right) .
$$

Applying here estimate

$$
m\left(e^{-1 / N}\right)=\exp \left\{L_{N}\left(e^{-1 / N}\right)\right\}=\exp \left\{L_{N}(1)\right\}\left(1+\left(L_{N}\left(e^{-1 / N}\right)-L_{N}(1)\right)+O\left(\rho^{2}\right)\right)
$$

we complete the proof of the theorem.

For $u>0$ we define

$$
E(u):=\exp \left\{2 \sum_{\substack{k=1 \\|\hat{f}(k)-1|>u}}^{n} \frac{|\hat{f}(k)-1|}{k}\right\} .
$$

Theorem 7. There exists such a constant $\eta=\eta\left(d^{-}, d^{+}\right)$that for any $u \leqslant \eta$ we have

$$
\left|M_{n} p_{n}{ }^{-1}\right| \ll\left|\exp \left\{L_{n}(1)\right\}\right|(E(u))^{d^{+}},
$$

Proof. Given a sequence of complex numbers $\hat{f}(j), j \geqslant 1$ and a positive number $u>0$ we can construct a new sequence $\hat{f}_{u}(j)$ defined as

$$
\hat{f}_{u}(j)= \begin{cases}1, & \text { if }|\hat{f}(j)-1|>u \\ \hat{f}(j), & \text { if }|\hat{f}(j)-1| \leqslant u\end{cases}
$$

We will denote the corresponding generating function of quantities $M_{k}^{(u)}$ corresponding to sequence of $\hat{f}_{u}(j)$ as

$$
M_{u}(z):=\exp \left\{\sum_{j=1}^{\infty} d_{j} \frac{\hat{f}_{u}(j)}{j} z^{j}\right\}=\exp \left\{L_{n}^{(u)}(z)\right\} p(z)=\sum_{k=0}^{\infty} M_{k}^{(u)} z^{k}
$$

where

$$
L_{n}^{(u)}(z)=\sum_{j=1}^{n} d_{j} \frac{\hat{f}_{u}(j)-1}{j} z^{j}=\sum_{\substack{j \leqslant n \\|\hat{f}(j)-1| \leqslant u}} d_{j} \frac{\hat{f}(j)-1}{j} z^{j}
$$


It is clear that the generating functions $M(z)$ and $M_{u}(z)$ are related by identity

$$
M(z)=M_{u}(z) \exp \left\{\sum_{|\hat{f}(j)-1|>u} d_{j} \frac{\hat{f}(j)-1}{j} z^{j}\right\} .
$$

The above identity leads to the relation between the coefficients in the Taylor expansion of the corresponding functions

$$
M_{n}=\sum_{k=0}^{n} M_{k}^{(u)} m_{n-k}^{(u)}
$$

where $m_{k}^{(u)}$ are the coefficients in the Taylor expansion of the generating function

$$
m^{(u)}(z)=\sum_{j=0}^{\infty} m_{j}^{(u)} z^{j}=\exp \left\{\sum_{|\hat{f}(j)-1|>u} d_{j} \frac{\hat{f}(j)-1}{j} z^{j}\right\} .
$$

Differentiating $m^{(u)}(z)$ one can easily see that $m_{k}^{(u)}$ satisfy the recurrent relationship

$$
m_{j}^{(u)}=\frac{1}{j} \sum_{\substack{1 \leqslant k \leqslant j \\|\hat{f}(k)-1|>u}} d_{k}(\hat{f}(k)-1) m_{j-k}^{(u)},
$$

which is true for all $j \geqslant 1$. Hence

$$
\begin{aligned}
\left|m_{j}^{(u)}\right| & =\left|\frac{1}{j} \sum_{\substack{1 \leqslant k \leqslant j \\
|\hat{f}(k)-1|>u}} d_{k}(\hat{f}(k)-1) m_{j-k}^{(u)}\right| \leqslant \frac{2 d^{+}}{j} \sum_{k=0}^{\infty}\left|m_{k}^{(u)}\right| \\
& \leqslant \frac{2 d^{+}}{j} \exp \left\{d^{+} \sum_{|\hat{f}(\ell)-1|>u} \frac{|\hat{f}(\ell)-1|}{\ell}\right\}=\frac{2 d^{+}}{j} E^{d^{+} / 2}(u) .
\end{aligned}
$$

Clearly, the newly formed sequence $\hat{f}_{u}(j)$ has a property that $\left|\hat{f}_{u}(j)-1\right| \leqslant u$ for all $j \geqslant 1$. Therefore if we assume that $u \leqslant \eta \leqslant \delta\left(d^{-}, d^{+}, \infty\right)$ then since $\rho(\infty) \leqslant u$ the conditions of the Proposition 3 will be satisfied, which gives us the estimate $M_{k}^{(u)}=p_{k} m^{(u)}\left(e^{-1 / k}\right)(1+O(u))$. We can now use this asymptotic of $M_{k}^{(u)}$ to estimate the right hand side of the identity (35) expressing $M_{n}$ in terms 
of $M_{j}^{(u)}$ and $m_{j}^{(u)}$ and obtain

$$
\begin{aligned}
\left|M_{n}\right| & =\sum_{k=0}^{n}\left|M_{k}^{(u)} m_{n-k}^{(u)}\right| \\
& \ll E^{d^{+} / 2}(u) \sum_{k \leqslant n / 2} \frac{p_{k}\left|m^{(u)}\left(e^{-1 / k}\right)\right|}{n-k}+\left|m^{(u)}\left(e^{-1 / n}\right)\right| p_{n}\left(\sum_{k=0}^{\infty}\left|m_{k}^{(u)}\right|\right) \\
& \ll E^{d^{+} / 2}(u)\left|m^{(u)}\left(e^{-1 / n}\right)\right|\left(\frac{1}{n} \sum_{k \leqslant n / 2} p_{k} \frac{\left|m^{(u)}\left(e^{-1 / k}\right)\right|}{\left|m^{(u)}\left(e^{-1 / n}\right)\right|}+p_{n}\right) \\
& \ll\left|\exp \left\{L_{n}^{(u)}(1)\right\}\right| E^{d^{+} / 2}(u)\left(\sum_{k \leqslant n / 2} p_{k}\left(\frac{n}{k}\right)^{u d^{+}}+p_{n}\right),
\end{aligned}
$$

here we have used the estimate $\frac{\left|m^{(u)}\left(e^{-1 / k}\right)\right|}{m^{(u)}\left(e^{-1 / n}\right) \mid}=\left|\exp \left\{\left(L_{k}^{(u)}\left(e^{-1 / k}\right)-L_{n}^{(u)}\left(e^{-1 / n}\right)\right)\right\}\right| \leqslant$ $\left(\frac{n}{k}\right)^{u d^{+}}$for $k \leqslant n$ and estimated the sum of $p_{k} k^{-u d^{+}}$by means of Lemma 9, assuming that $\eta$ fixed such that $u \leqslant \eta<\frac{d^{-}}{d^{+}}$. We finally obtain

$$
\left|M_{n}\right| \ll p_{n}\left|\exp \left\{L_{n}^{(u)}(1)\right\}\right| E^{d^{+} / 2}(u) \leqslant p_{n}\left|\exp \left\{L_{n}(1)\right\}\right| E^{d^{+}}(u) .
$$

Thus we have proven that the theorem holds with $\eta=\min \left\{\delta\left(d^{-}, d^{+}, \infty\right), d^{+} /\left(2 d^{-}\right)\right\}$.

Let us now find the generating function of the characteristic function $g(t)$ of the distribution of $h_{n}(\sigma)$. Notice that if $h_{n}(\sigma)$ is an additive function, then $\exp \left\{i t h_{n}(\sigma)\right\}$ is a multiplicative function of $\sigma$. Therefore

$g_{n}(t)=\mathbb{E} \exp \left\{i t h_{n}(\sigma)\right\}=\sum_{\sigma \in S_{n}} \exp \left\{i t h_{n}(\sigma)\right\} \nu_{n, d}(\sigma)=\frac{\sum_{\sigma \in S_{n}} d(\sigma) \exp \left\{i t h_{n}(\sigma)\right\}}{\sum_{\sigma \in S_{n}} d(\sigma)}$.

Thus we can apply our theorems for mean values of multiplicative functions for multiplicative function $f$ defined by $\hat{f}(k)=\exp \left\{i t \hat{h}_{n}(k)\right\}$ to analyze the asymptotic behavior of the characteristic function of $h_{n}(\sigma)$.

Proof of Theorem 4 Putting $\hat{f}(k)=e^{i t \hat{h}_{n}(k)}$ in Theorem 6 , for $|t| \leqslant \delta L_{n, p}^{-1 / p}$ we 
have

$$
\begin{aligned}
\phi_{n}(t) & :=\int_{-\infty}^{\infty} e^{i t x} d F_{n}(x) \\
& =e^{-i t A(n)} \exp \left\{L_{n}(1)\right\}\left(1+\sum_{j=1}^{n} d_{j} \frac{\hat{f}(j)-1}{j}\left(\frac{p_{n-j}}{p_{n}}-1\right)+O\left(\rho^{2}\right)\right) \\
& =e^{-t^{2} / 2+O\left(|t|^{3} L_{3, n}\right)}\left(1+C_{n} i t+O\left(|t|^{2} \sum_{j=1}^{n} d_{j} \frac{a_{n k}^{2}}{j}\left|\frac{p_{n-j}}{p_{n}}-1\right|\right)+O\left(|t|^{2} L_{n, p}^{2 / p}\right)\right) \\
& =e^{-t^{2} / 2}\left(1+C_{n} i t+O\left(|t|^{2}\left(1+|t|^{3}\right)\left(L_{n, p}^{2 / p}+L_{n, 3}+L_{n, 2}^{\prime}\right)\right)\right) .
\end{aligned}
$$

As in [8], from Theorem 7 we deduce the existence of some sufficiently small $c=c\left(d^{-}, d^{+}\right)$, that if $|t| \leqslant c L_{n, 3}^{-1}=: T$ then

$$
\left|\phi_{n}(t)\right| \ll e^{-c_{1} t^{2}}
$$

here $c_{1}=c_{1}\left(d^{-}, d^{+}\right)$is some fixed positive constant. Applying the generalized Esseen inequality (see for example [12]), we obtain

$\sup _{x \in \mathbb{R}}\left|F_{n}(x)-\Phi(x)+\frac{1}{\sqrt{2 \pi}} e^{-x^{2} / 2} C_{n}\right| \ll \int_{-T}^{T} \frac{\left|\phi_{n}(t)-e^{-t^{2} / 2}\left(1+C_{n} i t\right)\right|}{|t|} d t+\frac{1}{T}$.

Representing the integral on the right hand side of this inequality as a sum of integrals over the intervals $|t| \leqslant \delta L_{n, p}^{-1 / p}$ and $\delta L_{n, p}^{-1 / p}<|t| \leqslant T$ and applying estimates (36) and (37) in those intervals we obtain the proof of the theorem.

\section{References}

[1] G. J. Babu, E. Manstavičius, and V. Zacharovas. Limiting processes with dependent increments for measures on symmetric group of permutations. In Probability and number theory_Kanazawa 2005, volume 49 of Adv. Stud. Pure Math., pages 41-67. Math. Soc. Japan, Tokyo, 2007.

[2] L. Comtet. Advanced combinatorics. D. Reidel Publishing Co., Dordrecht, enlarged edition, 1974. The art of finite and infinite expansions.

[3] Ph. Flajolet and A. Odlyzko. Singularity analysis of generating functions. SIAM J. Discrete Math., 3(2):216-240, 1990. 
[4] W. Gontcharoff. Sur la distribution des cycles dans les permutations. C. R. (Doklady) Acad. Sci. URSS (N.S.), 35:267-269, 1942.

[5] G. Halász. Über die Mittelwerte multiplikativer zahlentheoretischer Funktionen. Acta Math. Acad. Sci. Hungar., 19:365-403, 1968.

[6] G. H. Hardy. Divergent Series. Oxford, at the Clarendon Press, 1949.

[7] J. Korevaar. Tauberian theory, volume 329 of Grundlehren der Mathematischen Wissenschaften [Fundamental Principles of Mathematical Sciences]. Springer-Verlag, Berlin, 2004. A century of developments.

[8] E. Manstavičius. The Berry-Esseen bound in the theory of random permutations. Ramanujan J., 2(1-2):185-199, 1998.

[9] E. Manstavičius. A Tauber theorem and multiplicative functions on permutations. In Number theory in progress, Vol. 2 (Zakopane-Kościelisko, 1997), pages 1025-1038. de Gruyter, Berlin, 1999.

[10] E. Manstavičius. Mappings on decomposable combinatorial structures: analytic approach. Combin. Probab. Comput., 11(1):61-78, 2002.

[11] E. Manstavičius. Additive and multiplicative functions on random permutations. Liet. Mat. Rink., 36(4):501-511, 1996.

[12] V. V. Petrov. Sums of independent random variables. Springer-Verlag, New York, 1975. Translated from the Russian by A. A. Brown, Ergebnisse der Mathematik und ihrer Grenzgebiete, Band 82.

[13] A. Tauber. Ein Satz aus der Theorie der unendlichen Reihen. Monatsh. Math. Phys., 8(1):273-277, 1897.

[14] V. Zacharovas. Cesàro summation and multiplicative functions on a symmetric group. Liet. Mat. Rink., 41(Special Issue):140-148, 2001.

[15] V. Zacharovas. The convergence rate in CLT for random variables on permutations. In Analytic and probabilistic methods in number theory (Palanga, 2001), pages 329-338. TEV, Vilnius, 2002. 